\title{
Dilaton gravity with a boundary: from unitarity to black hole evaporation
}

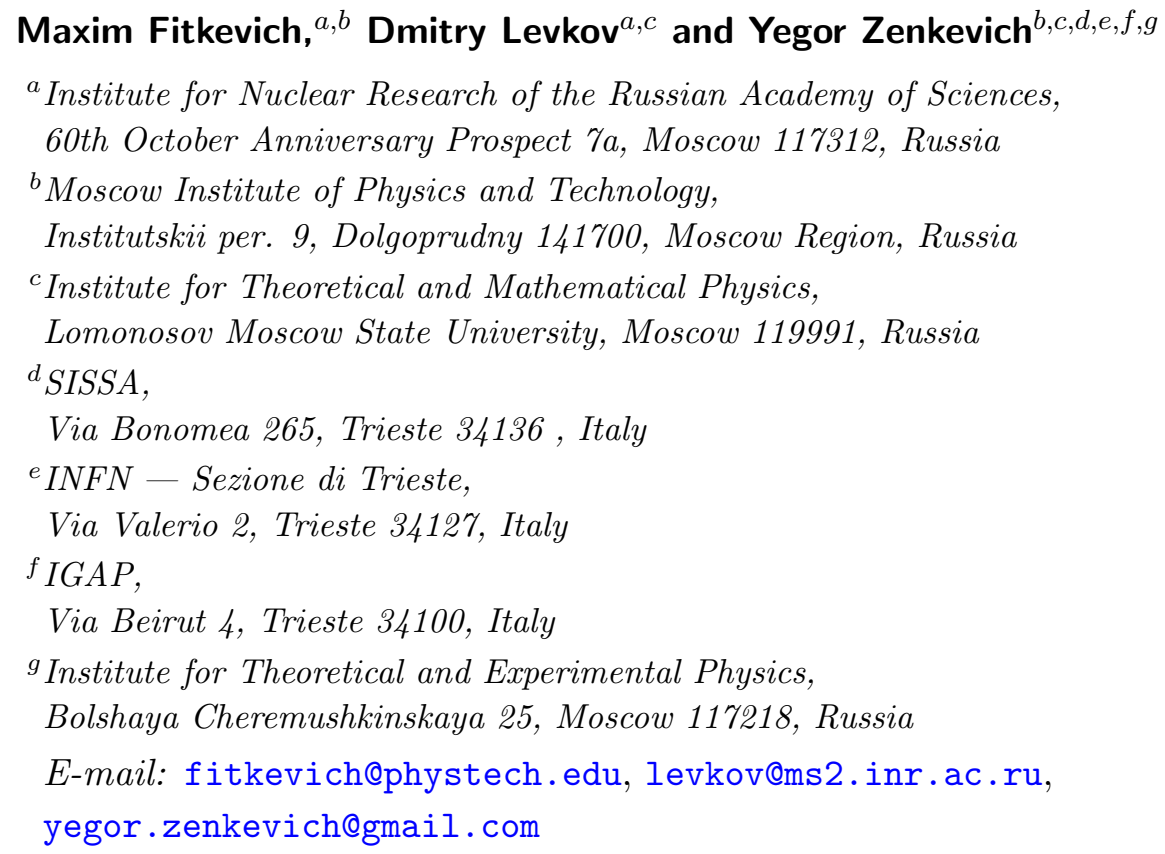

ABSTRACT: We point out that two-dimensional Russo-Susskind-Thorlacius (RST) model for evaporating black holes is locally equivalent - at the full quantum level - to flat-space Jackiw-Teitelboim (JT) gravity that was recently shown to be unitary. Globally, the two models differ by a reflective spacetime boundary added in the RST model. Treating the boundary as a local and covariant deformation of quantum JT theory, we develop sensible semiclassical description of evaporating RST black holes. Nevertheless, our semiclassical solutions fail to resolve the information recovery problem, and they do not indicate formation of remnants. This means that either the standard semiclassical method incorrectly describes the evaporation process or the RST boundary makes the flat-space JT model fundamentally inconsistent.

KeYwords: 2D Gravity, Black Holes, Models of Quantum Gravity

ArXiv EPrint: 2004.13745 


\section{Contents}

1 Introduction 1

2 From RST to JT 4

2.1 Weyl transformation 4

$\begin{array}{ll}2.2 & \text { Adding the boundary }\end{array}$

3 Evaporating black holes $\quad 8$

3.1 Semiclassical equations 8

$\begin{array}{lll}3.2 & \text { General properties of solutions } & 10\end{array}$

$\begin{array}{lll}3.3 & \text { Solvable deformation } & 12\end{array}$

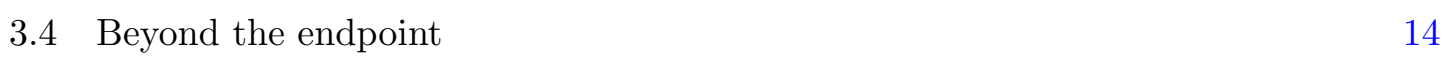

4 Information loss revisited $\quad 16$

$\begin{array}{lll}4.1 & \text { Endpoint singularity } & 16\end{array}$

$\begin{array}{lll}4.2 \text { Thunderpop } & 17\end{array}$

$\begin{array}{lll}4.3 & \text { Absence of remnants } & 19\end{array}$

4.4 Non-conservation of a global charge 20

$\begin{array}{lll}5 & \text { Discussion } & 21\end{array}$

A Deriving the semiclassical equations $\quad 23$

$\begin{array}{lll}\text { A.1 Solution in the bulk } & 23\end{array}$

A.2 Reflection laws 24

$\begin{array}{lll}\text { A.3 Energy conservation } & 25\end{array}$

A.4 Equations for solvable deformation 26

$\begin{array}{ll}\text { B Entanglement entropy } & 26\end{array}$

\section{Introduction}

Recently the simplest theory of two-dimensional dilaton gravity - flat-space JackiwTeitelboim (JT) model [1-3] — was quantized and its nontrivial, explicitly unitary $\mathcal{S}$-matrix was obtained $[4,5]$, see also $[6,7]$. This model displays so many features of full multidimensional gravity that one can hastily anticipate its application to the long-standing puzzles of black hole physics like information paradox [8-14], firewall proposal [15] (cf. [11]), or nonconservation of global charges [16-18]. However, the JT metric is flat on field equations, and all classical solutions in this theory are causally equivalent to empty two-dimensional spacetime, see figure 1(a). This precludes formation of authentic black holes with horizons 

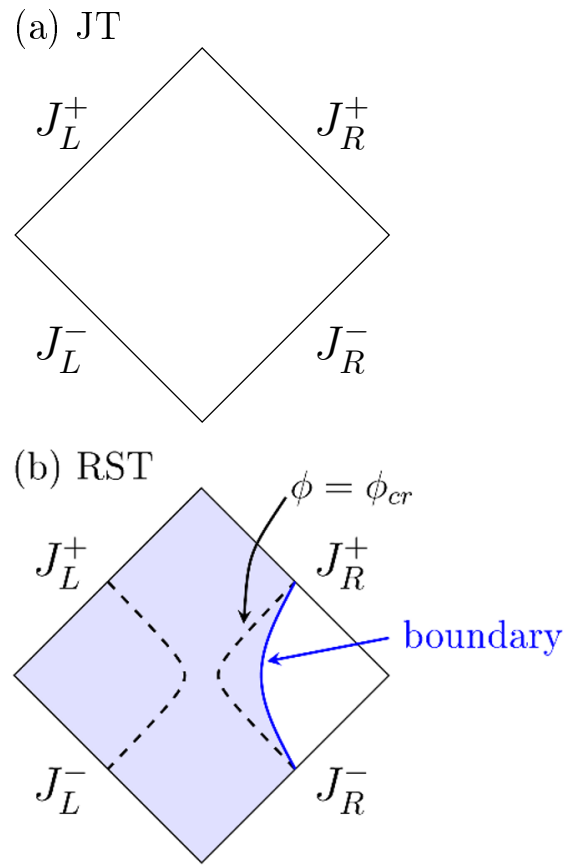

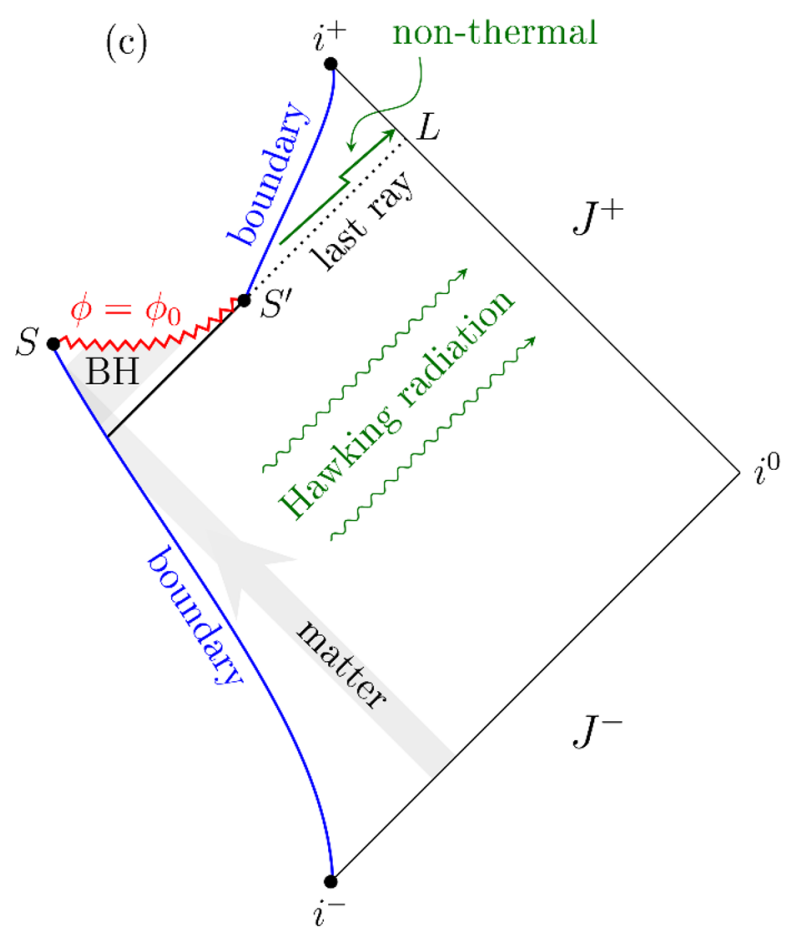

Figure 1. (a), (b) Causal structures of flat-space JT model and RST model. (c) Penrose diagram for the evaporating RST black hole.

and singularities. Introducing $N$ matter fields $\hat{f}_{i}(x)$, one can unitarily transfer information between the past and future JT infinities, but learn nothing about the black hole physics.

In this paper we consider the celebrated Russo-Susskind-Thorlacius (RST) model [19] which is specifically designed to describe evaporating black holes in two dimensions. We point out that this model is locally equivalent, at the full quantum level, to the unitary flat-space JT gravity. To establish the equivalence, one promotes the one-loop RST model to a full quantum theory: one adds RST counter-term [19] and $N$ matter fields $f_{i}(x)$ to the action of dilaton gravity [20], and then quantizes the resulting theory in a consistent way suggested by Strominger [21]. After that the path integrals of JT and RST models are related by the local Weyl transformation, ${ }^{1}$

$$
\hat{g}_{\mu \nu}=\mathrm{e}^{-2 \phi} g_{\mu \nu}, \quad \hat{\phi}=\mathrm{e}^{-2 \phi}+\frac{N \phi}{48 \pi}, \quad \hat{f}_{i}=f_{i},
$$

where $g_{\mu \nu}(x), \phi(x)$ and $f_{i}(x)$ are the RST metric, dilaton and matter fields, while $\hat{g}_{\mu \nu}(x)$, $\hat{\phi}(x)$ and $\hat{f}_{i}(x)$ are the fields in the flat-space JT model. Note that the change of variables (1.1) causes anomalous transformation of the functional measure which is important for the equivalence. In what follows we denote $Q^{2} \equiv N /(48 \pi)$.

Unfortunately, the transformation (1.1) is not defined globally. Indeed, it is noninvertible near the critical value of the dilaton field $\phi_{c r}=-\frac{1}{2} \log \left(Q^{2} / 2\right)$ corresponding to

\footnotetext{
${ }^{1}$ Equivalence at the classical level was previously established in [3, 22]. Flatness of Weyl-transformed one-loop geometry was pointed out in [21]. Also, it is known that redifinition (1.1) of the dilaton field is required [19] to solve the RST model at the semiclassical level.
} 
$d \hat{\phi} / d \phi=0$. This is a problem because all finite-energy JT/RST solutions, e.g. the linear dilaton vacuum, have position-dependent dilaton reaching $\phi_{c r}$ at some critical curve (dashed line in figure 1(b)). The transformation from the healthy JT theory to the RST variables is singular at this curve. Early works observed [19, 23-25] that the semiclassical solutions describing evaporating RST black holes develop curvature singularities at $\phi=\phi_{c r}$, and this impedes quantization of the theory [26]. We show that these singularities disappear in full quantum theory after transforming to the JT terms.

It would be natural to equate the RST and flat-space JT models discarding singular parametrization by the RST fields. But that would degrade the spectacular RST black holes to fictitious objects ${ }^{2}$ hiding information under the fake singularities of the RST fields, cf. [28].

In this paper we explore another possibility suggested in the original works $[19,29,30]$. Namely, we deform the RST model: we introduce a reflective spacetime boundary along the line of constant dilaton $\phi(x)=\phi_{0}<\phi_{c r}$ and restrict all fields in the path integral to the submanifold $\phi \leq \phi_{0}$ (white region in figure 1(b)). This excludes the Weyl singularity from the physical domain and makes the model causally similar to the spherically-symmetric multidimensional gravity.

Unlike in the earlier studies, we have a solid tool for selecting sensible boundary conditions at $\phi=\phi_{0}$. Indeed, we first add the boundary to the healthy flat-space JT theory, making it a local, covariant, self-consistent, and weakly coupled deformation, and then transform to the RST terms. Moreover, since (1.1) is valid at the quantum level, we compute one-loop effective action [31] with correct boundary terms [32] in the quantum JT theory and then perform Weyl transformation. This gives one-loop RST boundary conditions which automatically satisfy all self-consistency criteria [33]. Our reflection laws at $\phi=\phi_{0}$ are similar to those in [30,34] but differ from the laws in [29, 35-37].

Once the self-consistent boundary conditions are found, we re-inspect information loss problem in the RST model. To this end we study, both analytically [38] and numerically, the semiclassical solutions extremizing the one-loop effective action. A typical high-energy solution is shown in figure 1(c). It still displays some of the undesirable features observed in the earlier studies [39]. In particular, $\phi(x)$ equals $\phi_{0}$ at three distinct lines: the timelike boundaries $i^{-} S$ and $S^{\prime} i^{+}$, and a spacelike curve $S S^{\prime}$. We cannot impose reflective boundary condition at $S S^{\prime}$, as it would imply strong violation of causality, but we still have to trim the spacetime along this line. Thus, $S S^{\prime}$ is an analog of black hole singularity in the RST model. The incoming matter irreversibly disappears behind this line, cf. [19].

We find that the spacetime of evaporating black hole can be continued into the future beyond the last ray $S^{\prime} L$. Indeed, the "singularity" line $\phi=\phi_{0}$ generically becomes timelike after some point $S^{\prime}$ — the endpoint of evaporation. Imposing the boundary conditions in that region, we obtain the branch $S^{\prime} i^{+}$of reflective boundary in figure 1(c). This makes the spacetime flat in the asymptotic future $i^{+}$. The price to pay is the jump of second metric derivatives at the last ray $S^{\prime} L$ leading to a small $\delta$-burst of negative energy along this ray ("thunderpop" in $[19,39])$. The latter inconsistency, however, appears due to sharp change of boundary conditions across the point $S^{\prime}$. Once "smearing" near $S^{\prime}$ is allowed,

\footnotetext{
${ }^{2}$ These objects are different from the JT analogs of black holes introduced in [27].
} 
the "thunderpop" becomes smooth.

Despite our effort to construct a sensible model, the semiclassical solution in figure 1(c) still indicates an apparent loss of quantum coherence by the evaporating black hole. The effect is caused by the singularity $S S^{\prime}$ irreversibly "eating" the pure quantum state of the incoming matter. We support this intuition by computing the entanglement entropy [4042] of the final state at the future null infinity $i^{0} i^{+}$. We demonstrate that large entropy of Hawking radiation at $i^{0} L$ cannot be compensated by the non-thermal radiation at $\mathrm{Li}^{+}$. Thus, initial pure state of matter transforms into a density matrix with nonzero entanglement, and unitarity is broken.

The same mechanism that ruins quantum coherence implies apparent non-conservation of global charges by the evaporating black holes [16-18]. Indeed, our model possesses a global shift charge, and we explicitly demonstrate that it disappears behind the black hole singularity $S S^{\prime}$.

To summarize, in this paper we try to make sense of the evaporating RST black holes by appealing to the healthy flat-space JT theory deformed by a boundary. Nevertheless, we observe apparent information loss inside these objects. Possible reasons for this failure will be discussed in section 5 .

\section{From RST to JT}

\subsection{Weyl transformation}

Two-dimensional Russo-Susskind-Thorlacius (RST) model [19] describes interaction of $N$ matter fields $f_{j}(x)$ with non-dynamical gravitational sector: metric $g_{\mu \nu}(x)$ and dilaton $\phi(x)$. The action of the model

$$
S_{\mathrm{RST}}=\int d^{2} x \sqrt{-g}\left[e^{-2 \phi}\left(R+4(\nabla \phi)^{2}+4 \lambda^{2}\right)-\frac{1}{2} \sum_{j=1}^{N}\left(\nabla f_{j}\right)^{2}-Q^{2} \phi R\right],
$$

includes the classical part representing CGHS dilaton gravity [20] and a quantum counterterm [19] — the last term in the integrand - providing exact solvability at one-loop level. The parameter $Q^{2} \equiv N /(48 \pi)$ is proportional to the number of scalar fields, while $\lambda$ sets the energy scale of the model.

It will be important for us that the above model has a family of classical black hole solutions extremizing the classical action (2.1) without the counter-term, see [20]. These objects evaporate once quantum corrections are taken into account. However, naive description of the evaporation reveals a pathology [21] that has to be cured by choosing a proper quantization procedure.

Usually, one quantizes (2.1) by fixing the conformal gauge,

$$
g_{\mu \nu}=\mathrm{e}^{2 \rho(x)} \eta_{\mu \nu},
$$

and introducing the standard Faddeev-Popov ghosts [32] $b_{\mu \nu}$ and $c^{\mu}$ with $b_{\mu \nu}-b_{\nu \mu}=b_{\mu}^{\mu}=0$ and action $S_{g h}=-\int d^{2} x \sqrt{-g} b_{\mu \nu} \nabla^{\mu} c^{\nu}$. This gives path integral

$$
Z_{\mathrm{RST}}^{\text {naive }}=\int[d \rho d \phi d b d c d f]_{\rho} \mathrm{e}^{i S_{\mathrm{RST}}[\rho, \phi, f]+i S_{g h}[b, c]},
$$


where all functional measures depend on the metric scale factor $\rho(x)$ due to the Weyl anomaly. Now, we can evaluate eq. (2.3) in the one-loop approximation. To this end we integrate out quadratic fluctuations of all fields in some fixed background - say, the

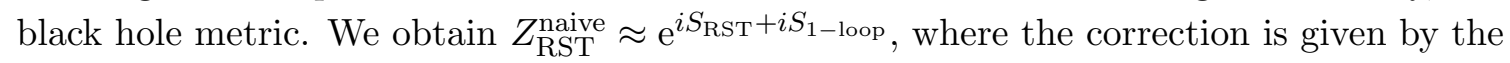
non-local Polyakov action [31],

$$
S_{1-\text { loop }}=-\frac{c}{96 \pi} \int d^{2} x d^{2} x^{\prime} \sqrt{g g^{\prime}} R \square^{-1}\left(x, x^{\prime}\right) R^{\prime} .
$$

In eq. (2.4) we introduced the Green's function $\square^{-1}$ of the d'Alembertian and denoted the total central charge of all fields by $c=N-24$. Extremizing the effective action (2.1), (2.4) with respect to the background metric and fields, one obtains one-loop semiclassical equations, with solutions describing evaporating black holes, see [20, 43, 44] and [19, 30, 36].

The problem is that the Hawking flux from these objects is always proportional [20] to the factor in front of the one-loop action (2.4) - the total central charge $c=N-24$. The latter, however, receives contributions from the entire field content of the model: $N$ from matter fields, +2 from non-dynamical fields $\phi$ and $\rho$, and -26 from ghosts. This means that at $N<24$ the black holes emit mainly ghosts and the total energy flux is negative. At larger $N$ the total flux, though positive, is not proportional to the number of dynamical fields $f_{j}$.

One may wonder, why Hawking evaporation of unphysical Faddeev-Popov ghosts is included in eq. (2.4). But this effect is unavoidable! Indeed, in Heisenberg picture the ghost operators $b_{\mu \nu}$ and $c^{\mu}$ satisfy causal equations in the black hole background, just like the ordinary fields. As a consequence, their positive- and negative-frequency components get mixed during evolution between the horizon and asymptotic infinity. Then the same Heisenberg vacuum that is ghost-free at the horizon, automatically contains a flux of ghosts at infinity. This suggests that eq. (2.3) is physically inconsistent.

Strominger proposed [21] a simple way to avoid this pathology. Namely, let us use the Weyl-transformed metric $\hat{g}_{\mu \nu}=\mathrm{e}^{-2 \phi(x)} g_{\mu \nu}$ with the scale factor $\rho-\phi$ in the functional measures of all non-dynamical fields $\rho, \phi, b, c$,

$$
Z_{\mathrm{RST}}=\int[d \rho d \phi d b d c]_{\rho-\phi}[d f]_{\rho} \mathrm{e}^{i S_{\mathrm{RST}}[\rho, \phi, f]+i S_{g h}[b, c]} .
$$

Below we will see that any classical solution in the model (2.1) has flat $\hat{g}_{\mu \nu}$. Then the unphysical fields are not emitted from the black holes. Indeed, loop contributions from these fields still produce the Polyakov action (2.4), but with the curvature $\hat{R}$ of $\hat{g}_{\mu \nu}$ replacing $R$. The effect of these contributions vanishes at $\hat{R}=0$ implying that the Hawking flux in eq. (2.5) is strictly proportional to the number $N$ of dynamical fields.

In what follows we use eq. (2.5) as a natural quantization of the RST model and do not consider alternative prescriptions.

Let us now argue that the quantum RST model is locally equivalent to the quantum flat-space JT theory [1-3]. To this end we rewrite the path integral (2.5) in variables (1.1) taking into account Weyl transformation law of the functional measure [32, 45],

$$
[d f]_{\rho}=[d f]_{\hat{\rho}} \exp \left\{\frac{i N}{24 \pi} \int d^{2} x \sqrt{-\hat{g}}\left[(\hat{\nabla} \phi)^{2}+\phi \hat{R}\right]\right\},
$$


where the new metric $\hat{g}_{\mu \nu}$ with scale factor $\hat{\rho} \equiv \rho-\phi$ is used everywhere in the right-hand side. We obtain,

$$
Z_{\mathrm{RST}}=\int[d \hat{\rho} d \hat{\phi} d b d c d \hat{f}]_{\hat{\rho}} \mathrm{e}^{i S_{J T}[\hat{\rho}, \hat{\phi}, \hat{f}]+i S_{g h}[b, c]}=Z_{J T},
$$

where

$$
S_{J T}=\int d^{2} x \sqrt{-\hat{g}}\left(\hat{\phi} \hat{R}+4 \lambda^{2}-\frac{1}{2} \sum_{j=1}^{N}\left(\hat{\nabla} \hat{f}_{j}\right)^{2}\right)
$$

is the action of the flat-space JT model with metric $\hat{g}_{\mu \nu}$, dilaton $\hat{\phi}$ and matter fields $\hat{f}_{j}$, see $[1-3,46]$.

The equivalence (2.7) is very natural. One can check [3] that the classical part of the RST action is related to the classical flat-space JT gravity by the transformation ${ }^{3}$ (1.1). Thus, any quantization on the RST side can be performed in JT terms and vice versa. Moreover, the RST counter-term in eq. (2.1) was originally introduced [19] to extend the symmetry $\hat{\phi} \rightarrow \hat{\phi}+$ const to one-loop level. This shift symmetry is manifest in (2.8), but nonlinearly realized in the RST terms. It is not a wonder that a consistent quantization preserving the symmetry reproduces the quantum flat-space JT model.

We summarize that the quantum RST model defined in (2.5) is a disguised version of flat-space JT gravity. Note that the JT metric $\hat{g}_{\mu \nu}$ is flat on the classical field equations because variation of (2.8) with respect to $\hat{\phi}$ gives $\hat{R}=0$. This property guarantees that the Hawking flux of unphysical fields is zero.

In what follows we will strongly rely on the fact $[4,5]$ that the flat-space JT gravity is a healthy quantum theory with unitary $\mathcal{S}$-matrix. This implies, in particular, that the RST model remains local and diffeomorphism-invariant after distortion of the functional measures performed in (2.5). Indeed, inverse Weyl transformation to eq. (2.6),

$$
[d \rho d \phi d b d c]_{\rho-\phi}=[d \rho d \phi d b d c]_{\rho} \exp \left\{\frac{i}{\pi} \int d^{2} x \sqrt{-g}\left[R \phi-(\nabla \phi)^{2}\right]\right\},
$$

gives path integral with canonical functional measures and new local counter-terms in the action.

\subsection{Adding the boundary}

Let us explicitly show that the transformation between the JT and RST models cannot be performed globally. Consider the classical JT vacuum in flat light-cone coordinates $(u, v)=$ $(t-x, t+x)$,

$$
\hat{g}_{\mu \nu}=\eta_{\mu \nu}, \quad \hat{\phi}=-\lambda^{2} u v .
$$

The dilaton $\hat{\phi}$ takes arbitrary values in this two-dimensional spacetime. On the other hand, the function $\hat{\phi}(\phi)$ in eq. (1.1) is bounded from below by the critical value $\hat{\phi}_{c r}$ given in the Introduction: $\hat{\phi}(\phi) \geq \hat{\phi}_{c r}$. Thus, the transformation (1.1) cannot be performed at $\hat{\phi}<\hat{\phi}_{c r}$ i.e. in the region between the dashed lines in figure 1(b). The latter lines are the true singularities of the RST fields.

\footnotetext{
${ }^{3}$ In the classical case one omits the second term in the expression for $\hat{\phi}(\phi)$.
} 
To remedy the RST model, we introduce a reflective spacetime boundary along the line of constant dilaton field $\phi=\phi_{0}, \phi_{0}<\phi_{c r}$. To this end we restrict all fields in the path integral (2.5) to the submanifold $\phi \leq \phi_{0}$ (the rightmost region in figure $1(\mathrm{~b})$ ) and add the boundary term to the action [38, 47],

$$
S_{\mathrm{RST}, \mathrm{b}}=\int_{\phi=\phi_{0}} d \tau\left[2 K\left(e^{-2 \phi_{0}}-Q^{2} \phi_{0}\right)-\mu\right],
$$

where $\tau$ is the proper time at $\phi=\phi_{0}$ and $K=\nabla_{\mu} n^{\mu}$ is the extrinsic curvature computed with the outer normal $n^{\mu}$. Expression (2.11) includes the Gibbons-Hawking term [48, 49] for the curvature part of the action (2.1) and a negative "mass" $\mu=-2 \lambda\left(2 e^{-2 \phi_{0}}+Q^{2}\right)$. We will see that the latter parameter stabilizes the position of the boundary in vacuum, cf. [38]. As before, $Q^{2} \equiv N /(48 \pi)$ appears in front of all counter-terms.

Note that the boundary makes the RST model weakly coupled, and the parameter $\mathrm{e}^{2 \phi_{0}} \ll 1$ controls semiclassical expansion. Indeed, the change of variables $\phi^{\prime} \equiv \phi-\phi_{0}$ and $f_{j}^{\prime} \equiv \mathrm{e}^{\phi_{0}} f_{j}$ brings $\mathrm{e}^{-2 \phi_{0}}$ in front of the action (2.1), (2.11) and makes all counter-terms proportional to $Q^{2} \mathrm{e}^{2 \phi_{0}} \sim N \mathrm{e}^{2 \phi_{0}}$. Below we are interested in the regime

$$
\mathrm{e}^{-2 \phi_{0}} \gg Q^{2} \gg 1
$$

where the quantum corrections are small and dominated by loops of matter fields.

To describe evaporating black holes in the RST model, we need to derive one-loop Polyakov action with correct boundary terms. It is uniquely fixed by the path integral (2.5). Note first that the boundary introduces local and covariant deformation of the JT action: performing the transformation (1.1) in eqs. (2.1), (2.11) and adding the correction ${ }^{4}(2.6)$, one obtains eq. (2.8) with the boundary term

$$
S_{J T, b}=\int_{\hat{\phi}=\hat{\phi}_{0}} d \hat{\tau}\left[2 \hat{\phi}_{0} \hat{K}-\mu \mathrm{e}^{\phi_{0}}\right]
$$

where $\hat{\phi}_{0}$ is related to $\phi_{0}$ by eq. (1.1). Next, we recall that Weyl transformation of the Polyakov action is fixed by the Wess-Zumino condition [32]. Namely, $\delta_{W} \hat{g}_{\mu \nu}=2 w(x) \hat{g}_{\mu \nu}$ should lead to

$$
\delta_{W} S_{J T, 1-\text { loop }}=\frac{c}{24 \pi}\left[\int d^{2} x \sqrt{-\hat{g}} \hat{R} w+2 \int_{\hat{\phi}=\hat{\phi}_{0}} d \hat{\tau} \hat{K} w\right],
$$

in any consistent quantum theory, e.g. in the flat-space JT gravity. From now on, we use $c=$ $N$ because non-dynamical fields will not contribute into the final Polyakov action anyway.

Solution of eq. (2.14) can be conveniently written in terms of an auxiliary field $\hat{\chi}(x)$ satisfying

$$
\hat{\square} \hat{\chi}=-Q \hat{R}, \quad \hat{n}^{\mu} \hat{\nabla}_{\mu} \hat{\chi}=2 Q \hat{K} .
$$

The Polyakov action is then

$$
S_{J T, 1-\text { loop }}=\int d^{2} x \sqrt{-\hat{g}}\left[-\frac{1}{2}(\hat{\nabla} \hat{\chi})^{2}+Q \hat{R} \hat{\chi}\right]+2 Q \int_{\hat{\phi}=\hat{\phi}_{0}} d \hat{\tau} \hat{K} \hat{\chi} .
$$

\footnotetext{
${ }^{4}$ One introduces the Gibbons-Hawking term $4 i Q^{2} \int d \hat{\tau} \phi \hat{K}$ in the exponent of (2.6) for consistency.
} 
One can explicitly check that it satisfies eq. (2.14) and coincides with eq. (2.4) in the bulk. Equations (2.15) simply mean that the action is extremal with respect to $\hat{\chi}$. Since we are going to solve the semiclassical field equations anyway, we will treat $\hat{\chi}$ on equal grounds with other fields.

Rewriting the effective action (2.8), (2.13), (2.16) in the RST terms (1.1), one obtains a consistent Polyakov correction to the RST model ${ }^{5}$

$$
S_{\mathrm{RST}, 1-\mathrm{loop}}=\int d^{2} x \sqrt{-g}\left[-\frac{1}{2}(\nabla \chi)^{2}+Q R \chi\right]+2 Q \int_{\phi=\phi_{0}} d \tau K \chi,
$$

where the auxiliary field $\chi \equiv \hat{\chi}+2 Q \phi$ extremizes the effective action.

To summarize, we have defined the quantum RST model, eqs. (2.5), (2.1), (2.11), in the most sensible way. First, we related it locally to the quantum flat-space JT theory $[1,2]$ with unitary $\mathcal{S}$-matrix $[4,5]$. Second, we regularized the singularity of the Weyl transformation with a dynamical boundary. This gave us the unique one-loop action (2.17). In what follows we describe black hole evaporation using this action.

\section{Evaporating black holes}

\subsection{Semiclassical equations}

Given the equivalence between the quantum RST and JT models, it is worth reanalyzing semiclassical evaporation of the RST black holes, cf. [19, 29, 30, 33, 34]. To this end we consider the one-loop effective action

$$
S=S_{\mathrm{RST}}+S_{\mathrm{RST}, b}+S_{\mathrm{RST}, 1-\mathrm{loop}}
$$

where the bulk action $S_{\mathrm{RST}}$, boundary term $S_{\mathrm{RST}, b}$, and one-loop correction $S_{\mathrm{RST}, 1-\text { loop }}$ are given by eqs. (2.1), (2.11), and (2.17), respectively. In what follows we use only one classical field $f=f_{1}$ keeping $f_{j}=0$ at $j \geq 2$. Recall that nevertheless, all fields fluctuate and contribute into $\chi$.

Let us review the semiclassical solutions extremizing the effective action; we leave details of their derivation to appendices A.1 and A.2. As usual, equations for the propagating fields $f, \chi$ simplify in the conformal frame (2.2) with $d s^{2}=-\mathrm{e}^{2 \rho} d u d v$, where we use the light-cone coordinates $u$ and $v$. General solution to these equations has the form,

$$
f=f_{\text {in }}(v)+f_{\text {out }}(u), \quad \chi=2 Q \rho(u, v)+\chi_{\text {in }}(v)+\chi_{\text {out }}(u),
$$

where $f_{\text {in,out }}$ are the incoming and outgoing wave packets, while $\chi_{\text {in,out }}$ are their quantum counterparts.

It is worth noting that eq. (2.2) does not completely fix the reparametrization invariance leaving residual conformal symmetry $u \rightarrow \tilde{u}(u), v \rightarrow \tilde{v}(v)$ [33]. Quantum wave packets transform nontrivially under this symmetry,

$$
\chi_{\mathrm{in}} \rightarrow \tilde{\chi}_{\mathrm{in}}(\tilde{v})=\chi_{\mathrm{in}}(v)+Q \log \left(\partial_{v} \tilde{v}\right), \quad \chi_{\text {out }} \rightarrow \tilde{\chi}_{\text {out }}(\tilde{u})=\chi_{\text {out }}(u)+Q \log \left(\partial_{u} \tilde{u}\right),
$$

\footnotetext{
${ }^{5}$ Of course, eq. (2.17) satisfies Wess-Zumino condition by itself, and one can derive it without resorting to the flat-space JT model. We imposed consistency requirement in explicitly sane terms to avoid confusion.
} 
see eq. (3.2). To explain the transformation law, we introduce the energy fluxes: the incoming flux

$$
T_{v v}(v)=\left(\partial_{v} f_{\text {in }}\right)^{2}+\left(\partial_{v} \chi_{\mathrm{in}}\right)^{2}+2 Q \partial_{v}^{2} \chi_{\mathrm{in}}
$$

and the outgoing flux $T_{u u}(u)$ - by the same expression with $v \rightarrow u$ and "in" $\rightarrow$ "out," see appendix A.1 for details. According to eq. (3.3), the fluxes have the standard conformal transformation laws, ${ }^{6}$ e.g.

$$
\tilde{T}_{\tilde{v} \tilde{v}}(\tilde{v})=\left(\partial_{v} \tilde{v}\right)^{-2}\left(T_{v v}(v)+2 Q^{2}\{\tilde{v} ; v\}\right)
$$

where the term with the Schwarzian $\{\tilde{v} ; v\}=\partial_{v}^{3} \tilde{v} / \partial_{v} \tilde{v}-\frac{3}{2}\left(\partial_{v}^{2} \tilde{v}\right)^{2} /\left(\partial_{v} \tilde{v}\right)^{2}$ comes from the quantum field $\chi_{\text {in }}$. As expected, this term is proportional to the total central charge $Q^{2} \propto N$.

Now, recall the correspondence (1.1) with the flat-space JT model. Since the JT metric is flat, $\hat{R}=0$, one can introduce flat coordinates with $\hat{g}_{\mu \nu}=\eta_{\mu \nu}$. In RST terms this corresponds to choosing the "Kruskal" gauge with

$$
\rho=\phi
$$

in eq. (2.2). In the model with a boundary the "Kruskal" coordinates $u$ and $v$ have semi-infinite ranges $-\infty<u<0$ and $0<v<+\infty$, see figure 1(b) and appendix A.1. Importantly, equations for the gravitational sector simplify in these coordinates giving

$$
\hat{\phi}=e^{-2 \phi}+Q^{2} \phi=-\lambda^{2} u v+g(v)+h(u),
$$

where

$$
\begin{aligned}
& g(v)=\frac{1}{2} \int_{0}^{v} d v^{\prime} \int_{v^{\prime}}^{\infty} d v^{\prime \prime}\left(T_{v v}\left(v^{\prime \prime}\right)+\frac{Q^{2}}{\left(v^{\prime \prime}\right)^{2}}\right)-\frac{Q^{2}}{2} \log (\lambda v), \\
& h(u)=-\frac{1}{2} \int_{-\infty}^{u} d u^{\prime} \int_{-\infty}^{u^{\prime}} d u^{\prime \prime}\left(T_{u u}\left(u^{\prime \prime}\right)+\frac{Q^{2}}{\left(u^{\prime \prime}\right)^{2}}\right)-\frac{Q^{2}}{2} \log (-\lambda u)
\end{aligned}
$$

are the second primitives of the energy fluxes.

Let us define the RST vacuum as a solution with $f_{\text {in }}=f_{\text {out }}=0$,

$$
\chi_{\mathrm{in}}=Q \log (\lambda v), \quad \chi_{\mathrm{out}}=Q \log (-\lambda u), \quad \phi=-\frac{1}{2} \log \left(-\lambda^{2} u v\right) .
$$

This configuration does not look like a vacuum in the "Kruskal" coordinates. However, transforming it via eqs. (3.3), (3.5) to the uniformly accelerating frame with respect to $u$ and $v$,

$$
\bar{u}=-\frac{1}{\lambda} \log (-\lambda u), \quad \bar{v}=\frac{1}{\lambda} \log (\lambda v) .
$$

one finds explicitly flat empty spacetime with $d s^{2}=-d \bar{u} d \bar{v}, \phi=\lambda(\bar{u}-\bar{v}) / 2, f=\chi=0$, and $\bar{T}_{\bar{u} \bar{u}}=\bar{T}_{\bar{v} \bar{v}}=0$. Thus, from the viewpoint of the quantum JT model $u$ and $v$ are flat coordinates and the RST vacuum (3.10) is a Rindler state. For the semiclassical RST

\footnotetext{
${ }^{6}$ Note that $T_{v v}$ and $T_{u u}$ are not the components of a reparametrization-covariant tensor, see their definition in appendix A.1.
} 
model, on the contrary, the RST vacuum (3.10) is the only empty flat solution and $\bar{u}=\bar{t}-\bar{x}$, $\bar{v}=\bar{t}+\bar{x}$ are flat coordinates. Note that the vacuum fluxes are negative in the "Kruskal" gauge: $T_{v v}=-Q^{2} / v^{2}$ and $T_{u u}=-Q^{2} / u^{2}$. They are subtracted in the integrands of eqs. (3.8) and (3.9) for convergence.

In what follows we describe the RST boundary $\phi=\phi_{0}$ by a function $u=U(v)$. We derive the reflection laws on this boundary in appendix A.2 by extremizing the effective action (3.1) with respect to the boundary values of the matter fields. This gives,

$$
f_{\text {out }}(U(v))=f_{\text {in }}(v), \quad \chi_{\text {out }}(U(v))=\chi_{\text {in }}(v)+Q \log \left(\partial_{v} U(v)\right),
$$

implying that the boundary acts precisely like a conformal transformation from $v$ to $u=$ $U(v)$, cf. eq. (3.3). The reflection laws relate the energy fluxes,

$$
T_{u u}(U(v))=\left(\partial_{v} U\right)^{-2}\left(T_{v v}(v)+2 Q^{2}\{U ; v\}\right) .
$$

cf. eq. (3.5). Note that the boundary condition (3.13) generically appears in all models with moving mirrors, cf. $[30,50,51]$, where the first term conserves classical energy in the rest frame of the mirror and the Schwarzian represents quantum particle production.

Equation of motion for the boundary $u=U(v)$ is obtained by recalling that $\phi=\phi_{0}$ along this line,

$$
\partial_{v} U=e^{2 \phi_{0}} \frac{q^{2}}{\lambda^{2}}\left(\partial_{v} g-\frac{Q^{2}}{2} \frac{\partial_{v}^{2} U}{\partial_{v} U}-\lambda^{2} U\right)^{2},
$$

where $q=\left(1+e^{2 \phi_{0}} Q^{2} / 2\right)^{-1}$, see appendix A.2. We stress that the derivation of this equation heavily relies on the reflection law (3.13); modification of the latter changes $U(v)$ as well. Terms with $Q^{2}$ in eq. (3.14) represent quantum corrections. They are small in the semiclassical regime $Q^{2} \ll \mathrm{e}^{-2 \phi_{0}}$ because after rescaling $f=\mathrm{e}^{-\phi_{0}} f^{\prime}, \chi=Q \chi^{\prime}$, and $U=\mathrm{e}^{-2 \phi_{0}} U^{\prime}$ equation (3.14) involve $Q$ and $\phi_{0}$ in the combination $Q^{2} \mathrm{e}^{2 \phi_{0}} \ll 1$.

Solving eq. (3.14) for the RST vacuum (3.10), one obtains,

$$
U(v)=-e^{-2 \phi_{0}} /\left(\lambda^{2} v\right) .
$$

Thus, the vacuum boundary is static, $\bar{x}(\bar{t})=-\phi_{0} / \lambda$, cf. eq. (3.11).

In what follows we solve the Cauchy problem in the semiclassical RST model. Namely, we prepare the finite-energy incoming wave packets $f_{\text {in }}(v)$ keeping the initial quantum field in vacuum, $\chi_{\mathrm{in}}=Q \log (\lambda v)$. Computing the incoming flux (3.4), (3.8), we solve the equation of motion (3.14) for $U(v)$. Then the reflection law (3.13) determines the outgoing flux $T_{u u}$, while eqs. (3.9), (3.7) fix the spacetime geometry $\rho=\phi$.

\subsection{General properties of solutions}

To warm up, we explore the semiclassical solutions numerically. We fix the incoming wave packet,

$$
\left(\partial_{v} f_{\text {in }}\right)^{2}=A \cosh ^{-2}\left(\sigma\left(v-v_{0}\right)\right),
$$

keeping its quantum counterpart $\chi_{\text {in }}$ in vacuum (3.10). Here the parameters $A, \sigma$, and $v_{0}$ represent respectively the amplitude, width, and initial position of the wave packet. 


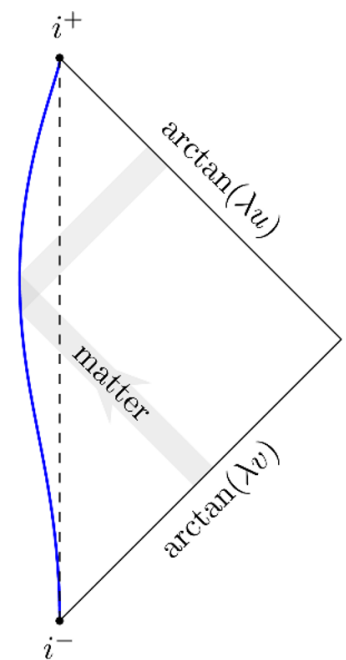

(a)

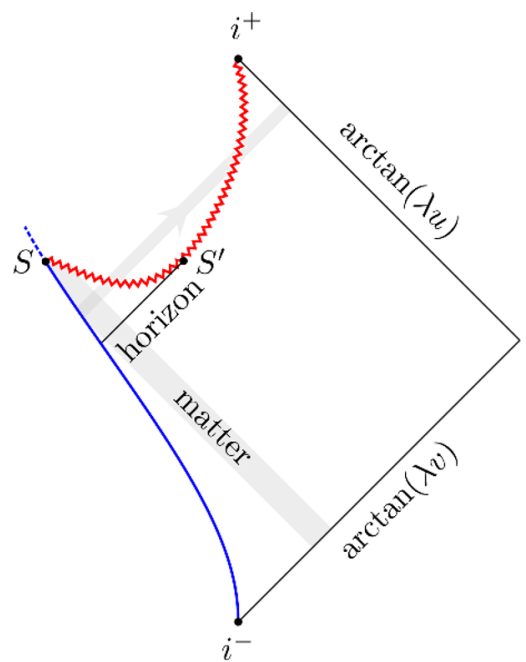

(b)

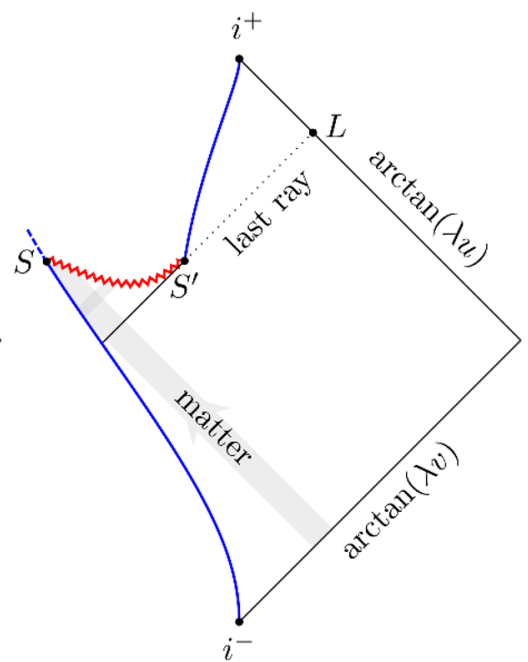

(c)

Figure 2. Numerical evolution of the wave packet (3.16) in the one-loop RST model; the respective parameters are $Q=0.5, \sigma=8.5 \lambda$, and $v_{0}=0.7 / \lambda$. For visualization purposes we set $\phi_{0}=0$. (a) Low-energy reflection at $A=2.5 \lambda^{2}$. (b) Black hole formation and evaporation at high energies: $A=10 \lambda^{2}$. (c) Natural extension of the solution (b).

Numerical integration of eq. (3.14) gives the boundary trajectories $U(v)$ at low and high energies of $f_{\text {in }}$, see the leftmost solid lines in figures $2(\mathrm{a})$ and $2(\mathrm{~b})$, respectively.

The low-energy solution in figure 2(a) describes trivial reflection of matter. Indeed, the respective boundary starts at $i^{-}$close to the vacuum solution $U \propto v^{-1}$ (dashed line), departs from it due to matter pressure and approaches it again at $i^{+}$. As a direct consequence, the reflected energy flux $T_{u u}$ in eq. (3.13) tends to the vacuum value at $u \rightarrow \pm \infty$, and the spacetime (3.7) becomes flat in the asymptotic past and future. We argue in appendix A.3 that the total energy of the outgoing matter in this case coincides with the energy of $f_{\text {in }}$.

The solution changes completely if the incoming energy exceeds certain value, see figure 2(b). In this case the boundary crosses the axis $u=0$ and continues growing. Numerically computing the dilaton in eq. (3.7), we discover another anomaly. Namely, the line $\phi(u, v)=\phi_{0}$ has two intersecting branches: the original timelike boundary $U(v)$ and the additional line $S i^{+}$with spacelike part $S S^{\prime}$. The semiclassical boundary conditions (3.12) are not satisfied along $\mathrm{Si}^{+}$, and one cannot enforce them without strongly violating causality. As a consequence, this line plays the role of a singularity in the one-loop RST model. Recall that we restrict the spacetime to the region $\phi<\phi_{0}$, below the line $S i^{+}$. Yet, the matter freely crosses this line and goes away, see eq. (3.2). At late times the singularity becomes timelike (part $S^{\prime} i^{+}$in figure 2(b)). We will consider this region in section 3.4.

Let us show that the salient features of solutions in figures $1(\mathrm{c})$ and $2(\mathrm{a}, \mathrm{b})$ are, in fact, generic. First, the RST vacuum is indeed stable, and all low-energy solutions correspond to reflection. Adding small perturbation $\delta \bar{u} \ll \lambda^{-1}$ to the vacuum solution, $U=-e^{-2 \phi_{0}}(1-$ 
$\lambda \delta \bar{u}) /\left(\lambda^{2} v\right)$, one arrives to the linearized equation,

$$
Q^{2} \partial_{\bar{v}}^{2} \delta \bar{u}+\left(\mathrm{e}^{-2 \phi_{0}}-Q^{2} / 2\right)\left(\lambda \partial_{\bar{v}}+\lambda^{2}\right) \delta \bar{u}=2 \partial_{\bar{v}} g+\lambda Q^{2},
$$

where the asymptotic RST coordinate $\bar{v}$ is used, eq. (3.11). Both fundamental solutions $\delta \bar{u} \propto \exp (i k \bar{v})$ of the left-hand side in this equation have $\operatorname{Im} k>0$ and therefore die off exponentially in the asymptotic future if $Q^{2} \mathrm{e}^{2 \phi_{0}}<2$, cf. (2.12). This means that the boundary shifted by the matter source in the right-hand side always returns back to the vacuum position, describing reflection. This regime holds if the incident energy is below some threshold.

Second, we prove that the spacelike "singularity" $\phi=\phi_{0}$ always forms in the limit of high matter flux, cf. figure 2(b). In this case the function $u=U(v)$ grows fast and crosses the axis $u=0$ due to large right-hand side in eq. (3.14). Define the apparent horizon ${ }^{7} u_{a}(v)$ as the boundary of the region where the curves of constant $\phi$ become space-like. Taking the derivative of eq. (3.7) along these curves and finding the point $d u / d v=0$, one obtains $u_{a}=\partial_{v} g(v) / \lambda^{2}$. Notably, the apparent horizon approaches the axis $u=0$ at $v \rightarrow+\infty$, see eq. (3.8). Then the growing boundary $u=U(v)$ intersects $u_{a}(v)$ at some point $S$ : $U\left(v_{s}\right)=u_{a}\left(v_{s}\right)$, and this is where the singularity appears. Indeed, $\partial_{u} \phi$ and $\partial_{v} \phi$ are zero at $S$, see eqs. (3.7) and (A.14). Hence, Taylor series expansion for $\phi(u, v)-\phi_{0}$ starts from quadratic terms near $S$. It will be convenient to relate the unknown coefficients of this expansion to the apparent velocity of the boundary $u_{1}=\partial_{v} U\left(v_{s}\right)$ using $\phi(U(v), v)=\phi_{0}$. Equation $\phi=\phi_{0}$ in the vicinity of the point $S$ takes the form,

$$
u_{1}^{2} T_{v v}\left(v-v_{s}\right)^{2}-\left(T_{v v}+4 \lambda^{2} u_{1}\right)\left(u-u_{s}\right)^{2}+4 \lambda^{2} u_{1}^{2}\left(u-u_{s}\right)\left(v-v_{s}\right)=0
$$

where $T_{v v}$ is the incoming flux at $v=v_{s}$ and we omitted higher-order terms in $v-v_{s}$ or $u-u_{s}$. Equation (3.18) has two solutions: the time-like boundary $u-u_{s}=u_{1}\left(v-v_{s}\right)$, where $u_{1}>0$ due to eq. (3.14), and the spacelike branch $u-u_{s}=-u_{1} T_{v v}\left(v-v_{s}\right) /\left(T_{v v}+4 \lambda^{2} u_{1}\right)$. This proves that the space-like singularity $\phi=\phi_{0}$ generically appears in high-energy solutions.

Note that the region behind the light-like "horizon" in figure 2 can be interpreted as the black hole interior, since matter in this region cannot escape the singularity.

\subsection{Solvable deformation}

Unfortunately, eq. (3.14) is not exactly solvable. The method to avoid this obstacle was suggested in [47]. To this end one imposes the Dirichlet boundary conditions ${ }^{8}$ on the auxiliary field $\chi$,

$$
\chi=\text { const } \quad \text { along } \quad u=U(v) .
$$

This modification is healthy at the one-loop level, but not in the full quantum approach: recall that the boundary condition for $\chi$ is fixed by the Wess-Zumino consistency condition [32]. Nevertheless, we will see that solutions in the deformed model (3.19) approximate well the original ones.

\footnotetext{
${ }^{7}$ Here we treat $\exp (-2 \phi)$ as an analog of the sphere area in the multidimensional gravity.

${ }^{8}$ More precisely, $\chi$ should be constant on every simply connected part of the boundary, cf. section 3.4.
} 
(a)

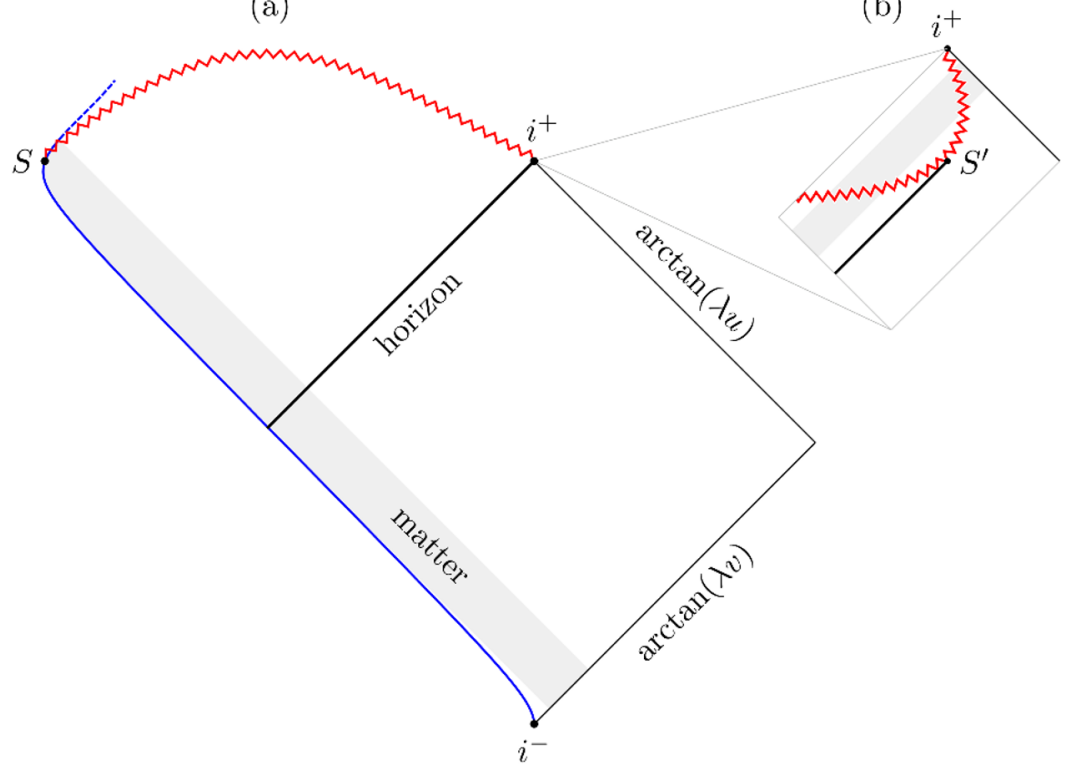

Figure 3. (a) Analytic solution (3.23), (3.24), (3.25) describing black hole formation and evaporation in the modified semiclassical model, cf. figure 2(b). For demonstration purposes we use $a=0$, $b=\lambda^{-1}, \phi_{0}=0$, and $Q=1$. (b) Zoom-in on the final stage of evaporation in figure (a).

The condition (3.19) gives the simplified reflection law for the quantum field, $\chi_{\text {out }}(U(v))=-\chi_{\text {in }}(v)+$ const, cf. eq. (3.12). As a consequence, the naive energymomentum tensors $T_{v v}^{(s)}=\left(\partial_{v} f_{\text {in }}\right)^{2}+\left(\partial_{v} \chi_{\text {in }}\right)^{2}$ and $T_{u u}^{(s)}$ reflect from the boundary classically, cf. [19],

$$
\left(\partial_{v} U\right)^{2} T_{u u}^{(s)}=T_{v v}^{(s)} .
$$

Physically, this means that the modification (3.19) switches off direct particle production by the accelerating boundary but leaves the field $\chi$ carrying the Hawking flux.

In appendix A.4 we derive equation for $U(v)$ in the solvable model [47],

$$
\partial_{v} U=e^{2 \phi_{0}} \frac{q^{2}}{\lambda^{2}}\left(\partial_{v} g+\frac{Q^{2}}{v}-\lambda^{2} U\right)^{2}
$$

where the incoming vacuum $\chi_{\mathrm{in}}=Q \log (\lambda v)$ for the quantum field is assumed. Comparing eqs. (3.21), (3.14), one finds that the modification replaces $\partial_{v}^{2} U / \partial_{v} U$ in the original equation with $-2 / v$. This is trustworthy in the limit of high energies when the boundary crosses the horizon before colliding with $f_{\text {in }}$. Indeed, the solution before the collision is $[30,47]$

$$
U \approx-\mathrm{e}^{-2 \phi_{0}} /\left(\lambda^{2} v\right)+c_{1}, \quad \text { with } \quad c_{1}=\lambda^{-2} \lim _{v \rightarrow 0}\left[\partial_{v} g(v)+Q^{2} /(2 v)\right] .
$$

With this behavior, equations (3.21) and (3.14) coincide everywhere outside the horizon. Apart from the high-energy limit, we expect that the model (3.19) will be useful at the qualitative level for any parameters.

Importantly, eq. (3.21) can be solved exactly [38]. Namely, changing the variables,

$$
U(v)=\frac{1}{\lambda^{2}}\left\{\partial_{v} g+\frac{Q^{2}}{v}-\frac{\mathrm{e}^{-2 \phi_{0}}}{q^{2}} W(v)\right\},
$$


one obtains a Riccati equation

$$
\left(\partial_{v} f_{\text {in }}\right)^{2}=-\frac{Q^{2}}{v^{2}}-2 \frac{\mathrm{e}^{-2 \phi_{0}}}{q^{2}}\left(\partial_{v} W+W^{2}\right)
$$

for the unknown $W(v)$. Alternatively, one can fix $W(v)$ and compute $U(v), f_{\text {in }}(v)$ by eqs. (3.23), (3.24). The nontrivial problem, however, is to find the $W$-ansatz that gives localized and positive-definite $\left(\partial_{v} f_{\text {in }}\right)^{2}$.

It was shown in ref. [38] that rational functions from a wide class satisfy the necessary requirements. The simplest ansatz is

$$
W=\frac{q}{v}+\frac{1}{v-c}-\frac{v-a}{(v-a)^{2}+b^{2}},
$$

where $a$ and $b$ are free real parameters and the constant

$$
c=a+\frac{1}{2(1-q)}\left[\sqrt{a^{2}+4 b^{2} q(1-q)}-a\right],
$$

is fixed by requiring the regularity of the incoming flux: the pole at $v=c$ in eq. (3.24) should be absent. Once this is fixed, $\left(\partial_{v} f_{\text {in }}\right)^{2}$ is a smooth and positive-definite function in a wide range of $a, b$. Besides, one can explicitly check that the asymptotic flux $\bar{T}_{\bar{v} \bar{v}}=\mathrm{e}^{2 \lambda \bar{v}}\left(\partial_{v} f_{\text {in }}\right)^{2}$ is localized i.e. vanishes exponentially at $\bar{v} \rightarrow \pm \infty$, see eq. (3.11).

Penrose diagram for the exact solution (3.23), (3.24), (3.25) is shown in figure 3. It has the same qualitative features as the original diagram in figure $2(\mathrm{~b})$, with main distinctions related to different choice of the incoming flux, cf. eqs. (3.24), (3.25) and (3.16). Note that using the method of ref. [38] one can construct an infinite number of multi-parameter solutions with different shapes of the incoming wave packets. The latter solutions can be classified using the Gaudin spin chain as an auxiliary tool.

\subsection{Beyond the endpoint}

At late times the singularity in figure 2(b) becomes timelike, cf. figure 3(b). The endpoint $S^{\prime}=\left(u_{e}, v_{e}\right)$ where this happens satisfies

$$
\hat{\phi}\left(u_{e}, v_{e}\right)=\hat{\phi}_{0}, \quad \partial_{v} \hat{\phi}\left(u_{e}, v_{e}\right) \equiv-\lambda^{2} u_{e}+\partial_{v} g\left(v_{e}\right)=0,
$$

where we used eq. (3.7).

Let us compute $u_{e}$ and $v_{e}$ in the quasi-stationary limit (2.12) when evaporation takes large asymptotic time $\bar{t}$ due to small $Q^{2}$. In this case $v_{e} \propto \mathrm{e}^{\lambda \bar{t}}$ is exponentially large and $u_{e}$ is small, ${ }^{9}$ see eq. (3.11). Thus, the incoming flux $T_{v v}\left(v_{e}\right)$ at the endpoint is already in vacuum implying

$$
g(v) \approx \frac{E_{\text {in }}}{2 \lambda}-\frac{Q^{2}}{2} \log (\lambda v) \quad \text { at } \quad v \sim v_{e}
$$

\footnotetext{
${ }^{9}$ The value of $\lambda u_{e}$ is visibly large in figure $2(\mathrm{~b})$ where $Q^{2} \sim \mathrm{e}^{-2 \phi_{0}}$ for visualization purposes. Typical black hole spacetimes at small $Q^{2}$ have $\lambda u_{e} \ll 1$ like in figure 3 .
} 
where we expressed the integral in eq. (3.8) in terms of the total energy $E_{\text {in }}$, see eq. (A.17) of appendix A.3. The other integral $h(u)$ is related to $g(v)$ by reflection from $U(v)$. Introducing the point $v_{\times}$where $U\left(v_{\times}\right)=0$, one obtains, $h\left(u_{e}\right) \approx \hat{\phi}_{0}-g\left(v_{\times}\right)$. This expression and eqs. (3.27), (3.28), (3.7) give the solution [19],

$$
u_{e} \approx-\frac{Q^{2}}{2 \lambda^{2} v_{e}}, \quad v_{e} \approx \frac{1}{\lambda} \exp \left\{1+\frac{E_{\text {in }}-2 \lambda g\left(v_{\times}\right)}{\lambda Q^{2}}\right\}
$$

which confirms that $v_{e}$ is indeed exponentially large at small $Q^{2}$. Indeed, at large energies when eq. (3.22) is approximately valid, $2 \lambda g\left(v_{\times}\right) \approx M_{c r}$, where $M_{c r}=2 \lambda \mathrm{e}^{-2 \phi_{0}}$ is the minimal black hole mass at $Q^{2} \rightarrow 0$, see [20,38]. We will see that $M_{c r}$ coincides with the black hole mass at the endpoint; it is smaller than $E_{\text {in }}$.

We have just shown explicitly that the endpoint (3.29) exists in generic quasi-stationary spacetimes of evaporating black holes. Beyond the endpoint the line $\phi=\phi_{0}$ is a naked timelike singularity. A natural way to cure this pathology is to impose reflective boundary conditions (3.13) at the timelike branch $S^{\prime} i^{+}$of the line $\phi=\phi_{0}$. In this case one solves eq. (3.14) for the second boundary $U_{2}(v)$ starting from $U_{2}\left(v_{e}\right)=u_{e}$ with some apparent velocity $\partial_{v} U_{2}\left(v_{e}\right)$, see figure $2(\mathrm{c})$. Since the incoming flux is already at vacuum, $U_{2}(v)$ trivially approaches the equilibrium trajectory $U_{2} \rightarrow-\mathrm{e}^{-2 \phi_{0}} /\left(\lambda^{2} v\right)$ at $v \rightarrow+\infty$, see discussion in section 3.2. Nevertheless, the resulting spacetime is special in two respects. First, it depends on the arbitrary parameter $\partial_{v} U_{2}\left(v_{e}\right)$. Second, it is non-analytic at the last ray $S^{\prime} L$ due to the jump of the boundary condition at $S^{\prime}$.

Importantly, the initial velocity $\partial_{v} U_{2}\left(v_{e}\right)$ of the new boundary is bounded from above. Indeed, the endpoint is a meeting place of $U_{2}(v)$ with the apparent horizon $\partial_{v} \hat{\phi}=0$, see eq. (3.27). In section 3.2 we learned that the line $\phi=\phi_{0}$ generically bifurcates at such points, with reflective boundary conditions broken along the additional "singular" branch. We therefore require that the singularity is not naked like in figure 4(a), but hides under $U_{2}(v)$. This gives

$$
0<\partial_{v} U_{2}\left(v_{e}\right)<-\frac{T_{v v}\left(v_{e}\right)}{2 \lambda^{2}} \approx \frac{Q^{2}}{4 \lambda^{2} v_{e}^{2}},
$$

where we solved eq. (3.18) and substituted the vacuum flux in the approximate equality. We will see that eq. (3.30) ruins the "remnant" explanation of the RST information paradox, cf. [28].

Now, consider the energy balance of the complete solution in figure 2(c). Using appendix A.3, we compute the total energy of black hole emission before the last ray,

$$
\begin{aligned}
E_{\text {out }, u<u_{e}} & \approx-2 \lambda h\left(u_{e}\right)-\lambda Q^{2} \log \left(-\lambda u_{e}\right) \\
& =E_{\text {in }}-M_{\text {cr }}-\lambda Q^{2} \log \left(Q^{2} \mathrm{e}^{2 \phi_{0}}\right)+O\left(Q^{2}\right),
\end{aligned}
$$

where we ignored $O\left(u_{e}\right)$ corrections, used eqs. (3.27), (3.7), (3.28), (3.29) and again introduced the minimal black hole mass $M_{\mathrm{cr}}=2 \lambda \mathrm{e}^{-2 \phi_{0}}$.

The outgoing energy (3.31) appears due to Hawking effect. Indeed, in the small vicinity of the horizon one can write $U(v) \approx \partial_{v} U\left(v_{\times}\right) \cdot\left(v-v_{\times}\right)$, where $U\left(v_{\times}\right)=0$. In terms of the asymptotic coordinate $\bar{u}$ this vicinity is a large region, since $\bar{u} \approx-\lambda^{-1} \log \left(\lambda v_{\times}-\mathrm{e}^{\lambda \bar{v}}\right)+$ 
const is singular at $v \rightarrow v_{\times}$. Using this $\bar{u}(\bar{v})$, one computes the reflected energy flux via eq. (3.13),

$$
\bar{T}_{\bar{u} \bar{u}} \approx \frac{\bar{T}_{\bar{v} \bar{v}} \mathrm{e}^{-2 \lambda \bar{u}}}{\lambda^{2} v_{\times}^{2}}+\lambda^{2} Q^{2}
$$

where the second term comes from the Schwarzian. With time, the stimulated emission in the first term dies off leaving the stationary thermal flux $\lambda^{2} Q^{2}=\lambda^{2} N /(48 \pi)$. Recall that the right-moving Bose gas with $N$ species and Hawking temperature $T_{H}=\lambda /(2 \pi)$ has

$$
\text { thermal flux }=N \int_{0}^{\infty} \frac{d k}{2 \pi} \frac{k}{e^{k / T_{H}}-1}=N \frac{\pi T_{H}^{2}}{12}=\lambda^{2} Q^{2}
$$

precisely the same as in eq. (3.32).

Note that although $h(u)$ is continuous at $u=u_{e}$, its derivative is not. Indeed, differentiating (3.7) along the boundaries $U(v)$ and $U_{2}(v)$ at $u=u_{e} \pm 0$, we find,

$$
u_{e} \partial_{u} h\left(u_{e}-0\right)=O\left(u_{e}\right), \quad u_{e} \partial_{u} h\left(u_{e}+0\right)=\lambda^{2} u_{e} v_{e}=-Q^{2} / 2,
$$

where eqs. (3.27), (3.29) were used. This jump reflects $\delta$-function singularity of the outgoing flux along the last ray - the thunderpop. Using eq. (A.17) of appendix A.3, we find that the energy of the thunderpop is negative [19],

$$
E_{\mathrm{th}-\mathrm{pop}}=\left.2 \lambda u_{e} \partial_{u} h\right|_{u_{e}-0} ^{u_{e}+0}=-\lambda Q^{2} .
$$

In section 4.2 we will remind that the thunderpop is an inevitable artifact of non-analytic sewing of two classical spacetimes along the last ray. It gives negligible energy contribution at small $Q^{2}$, so one can disregard it altogether.

In appendix A.3 we show that the total energy is conserved for the complete solution in figure 3(c) which starts from the RST vacuum in the past and arrives to it in the future, see also [47]. This means that the remaining energy

$$
E_{\text {out }, u>u_{e}}=M_{\text {cr }}+\lambda Q^{2} \log \left(Q^{2} \mathrm{e}^{2 \phi_{0}}\right)+O\left(Q^{2}\right)
$$

is emitted ${ }^{10}$ in a non-thermal way at $u>u_{e}$. Expression (3.35) is the mass of the critical black hole at the endpoint of evaporation, decaying afterwards.

In figure 4(b) we plot the outgoing energy flux for the numerical solution in figure 2(c). It displays all features observed above. Notably, it is not strictly positive-definite beyond the last ray, which is expected [52-54].

\section{Information loss revisited}

\subsection{Endpoint singularity}

At first glance, the semiclassical RST solutions look smooth - even their "singularities" are just the spacelike branches of the line $\phi=\phi_{0}$. Nevertheless, one runs into a trouble

\footnotetext{
${ }^{10}$ One can directly calculate it using eq. (A.17) of appendix A.3.
} 


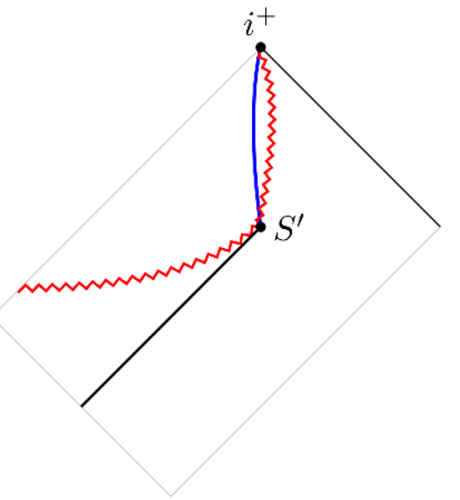

(a)

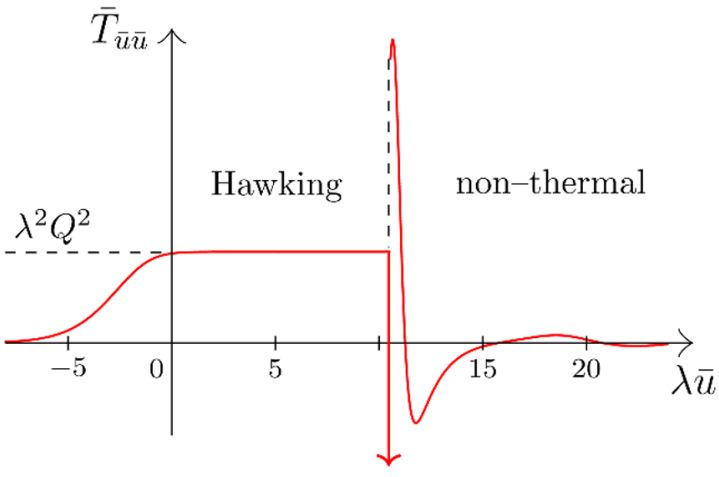

L: th-pop

(b)

Figure 4. (a) Solution with the second boundary violating eq. (3.30), cf. figure 2(c). (b) The asymptotic outgoing energy flux for the numerical solution in figure $2(\mathrm{c})$.

trying to apply these solutions to computation of the effective action (3.1). Indeed, the left border of the spacetime in figure 2(b) is not smooth but constructed from three time- and spacelike pieces (lines $i^{-} S, S S^{\prime}$, and $S^{\prime} i^{+}$). It is not clear how to evaluate the extrinsic curvature $K$ and therefore the Gibbons-Hawking term (2.11) at the angles $S$ and $S^{\prime}$ of these pieces.

One can try to regularize $S^{\prime}$ with a smooth curve $U_{\text {reg }}(v)$ going between the spacelike and timelike branches of $\phi=\phi_{0}$. Then the curve becomes light-like at some point $v_{0} \approx v_{e}$, with $U_{\text {reg }}(v)-U_{\text {reg }}\left(v_{0}\right) \propto\left(v-v_{0}\right)^{2}$ near it. Using appendix A.2, we evaluate the extrinsic curvature of $U_{\text {reg }}(v)$ in this region,

$$
K(\tau) \sim \frac{1}{3\left(\tau-\tau_{0}\right)}+\text { regular part }
$$

where $\tau$ is a proper distance along $U_{\text {reg }}$ at $v<v_{0}$ and a proper time at $v>v_{0}$, with $\tau=\tau_{0}$ corresponding to $v=v_{0}$. We see that the Gibbons-Hawking integral (2.11) diverges at the light-like point $\tau=\tau_{0}$, and there is no apparent way to regularize it, cf. [55, 56].

As a second try, we can leave the angles $S$ and $S^{\prime}$ as they are and simply ignore their Gibbons-Hawking contributions. But then arbitrary local counter-terms $S_{S}$ and $S_{S^{\prime}}$ depending on all RST fields at the respective points can be added to the effective action. Importantly, the point $S^{\prime}$ is visible to the distant observer. As a consequence, its counterterm sets boundary conditions for future evolution: the apparent initial velocity of $U_{2}(v)$ and quantum state of matter going along the last ray $S^{\prime} L$.

To summarize, the point $S^{\prime}$ is a naked singularity of the complete solution. It adds arbitrary parameters to the problem and therefore limits our understanding of evaporating black holes.

\subsection{Thunderpop}

In section 3.4 we have found the thunderpop - an outgoing $\delta$-flux carrying small negative energy (3.34) along the last ray, cf. [19]. It is harmless and can be ignored. There is a 


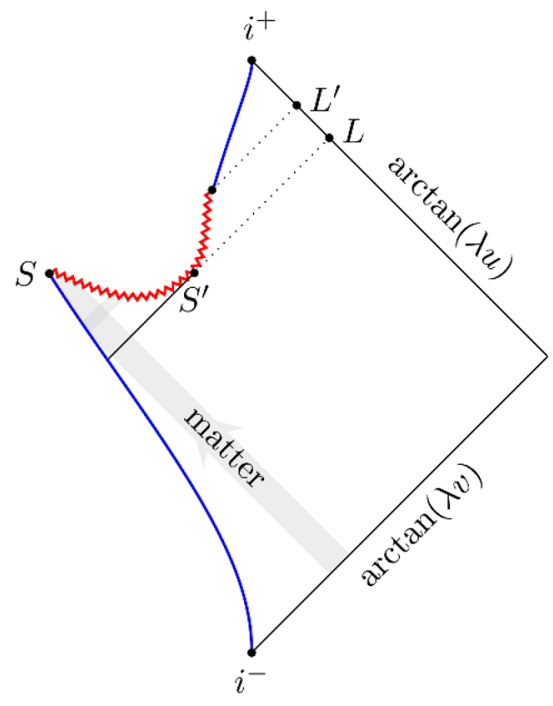

(a)

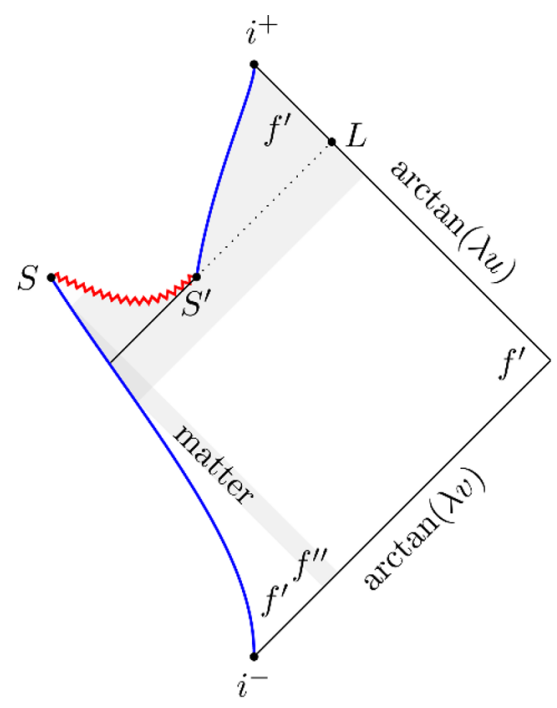

(b)

Figure 5. (a) Smearing the singularity $S^{\prime}$. (b) Global charge violation in the RST model.

belief in the literature $[39,56]$, however, that the last ray in causal one-loop models always turns into an infinite-energy "thunderbolt" singularity. Let us show, why this is not the case in our model.

For a start, we reproduce the argument of [39]. Consider the quantum correlator of one RST field, say, $f_{N}$. Initially, this field is in vacuum,

$$
\left\langle f_{N, \text { in }}\left(\bar{v}_{1}\right) f_{N, \text { in }}\left(\bar{v}_{2}\right)\right\rangle=-\frac{1}{4 \pi} \log \left|\bar{v}_{2}-\bar{v}_{1}\right|+\text { const } .
$$

The outgoing two-point function then follows from the reflection law (3.12),

$$
\begin{aligned}
\left\langle f_{N, \text { out }}\left(\bar{u}_{1}\right) f_{N, \text { out }}\left(\bar{u}_{2}\right)\right\rangle_{\text {reg }} & \equiv\left\langle f_{N, \text { out }}\left(\bar{u}_{1}\right) f_{N, \text { out }}\left(\bar{u}_{2}\right)\right\rangle-\left\langle f_{N, \text { out }}\left(\bar{u}_{1}\right) f_{N, \text { out }}\left(\bar{u}_{2}\right)\right\rangle_{\mathrm{vac}} \\
& =-\frac{1}{4 \pi}\left(\log \left|\bar{v}\left(\bar{u}_{2}\right)-\bar{v}\left(\bar{u}_{1}\right)\right|-\log \left|\bar{u}_{2}-\bar{u}_{1}\right|\right)+\mathrm{const},
\end{aligned}
$$

where we subtracted the vacuum correlator for regularity and introduced the boundary $\bar{v}(\bar{u})$ in asymptotic coordinates.

If the boundary is smooth, the regularized correlator (4.2) is finite in the limit $\bar{u}_{1} \rightarrow \bar{u}_{2}$ due to cancellation between the first and second terms. However, $\bar{v}(\bar{u})$ has a jump at the last ray $\bar{u}=\bar{u}_{e}$, see figure 2(c). As a consequence, the first term stays finite when $\bar{u}_{1}$ and $\bar{u}_{2}$ approach $\bar{u}_{e}$ from different sides, and the second term is divergent. This produces a strong non-integrable singularity in the energy flux at $\bar{u}=\bar{u}_{e}$,

$$
\left\langle\bar{T}_{\bar{u} \bar{u}}^{(N)}\left(\bar{u}_{e}\right)\right\rangle_{\text {reg }}=\lim _{\bar{u}_{2}, \bar{u}_{1} \rightarrow \bar{u}_{e}} \partial_{\bar{u}_{2}} \partial_{\bar{u}_{1}}\left\langle f_{N, \text { out }}\left(\bar{u}_{1}\right) f_{N, \text { out }}\left(\bar{u}_{2}\right)\right\rangle_{\mathrm{reg}} \sim \lim _{\bar{u}_{2}, \bar{u}_{1} \rightarrow \bar{u}_{e}} \frac{1}{4 \pi\left(\bar{u}_{2}-\bar{u}_{1}\right)^{2}},
$$

where we used regularization by point separation.

We have already argued, however, that the endpoint $S^{\prime}$ is a naked singularity in the one-loop model, and the quantum state of the fields $f_{i}$ leaving this point is not under 
control. Let us smear this point into a tiny timelike singularity $\phi=\phi_{0}$, see figure 5(a). Then $f_{N \text {, out }}$ is not fixed at $\bar{u}_{L}<\bar{u}<\bar{u}_{L^{\prime}}$. One can therefore continue the regularized correlator (4.2) into this region in an arbitrarily smooth way, matching it together with its first two derivatives to the correlators at $\bar{u}_{L}$ and $\bar{u}_{L^{\prime}}$. This will give finite energy-momentum tensor inside the interval $L L^{\prime}$. There is a restriction, however: the jump of the primitive $\partial_{u} h$ between $\bar{u}_{L}$ and $\bar{u}_{L^{\prime}}$ is fixed by the equations for the boundary, see eq. (3.33). This gives the total energy $E_{\mathrm{th}-\text { pop }} \approx-\lambda Q^{2}$ inside the "quantum" region $L L^{\prime}$. Notably, $E_{\text {th-pop }}$ in vanishingly small in the quasi-stationary limit (2.12) that we consider.

\subsection{Absence of remnants}

Let us make the information paradox explicit in our model. To this end we introduce geometric entropy $\Sigma_{\text {reg }}\left(\bar{u}_{1}, \bar{u}_{2}\right)$ characterizing entanglement of the outgoing fields $\left\{f_{i, \text { out }}\right\}$ inside the interval $\bar{u}_{1}<\bar{u}<\bar{u}_{2}$ with anything outside it [40]. In appendix B we evaluate the entropies of the Hawking quanta $\Sigma^{-} \equiv \Sigma_{\text {reg }}\left(-\infty, \bar{u}_{e}-0\right)$ before the last ray and of non-thermal radiation beyond the endpoint $\Sigma^{+} \equiv \Sigma_{\text {reg }}\left(\bar{u}_{e}+0,+\infty\right)$, see figure 2(c). For $N=48 \pi Q^{2}$ fields,

$$
\Sigma^{-}=-\left.4 \pi Q^{2} \log \frac{d \bar{v}}{d \bar{u}}\right|_{\bar{u}=\bar{u}_{e}-0}, \quad \Sigma^{+}=-\left.4 \pi Q^{2} \log \frac{d \bar{v}}{d \bar{u}}\right|_{\bar{u}=\bar{u}_{e}+0},
$$

where $\bar{v}(\bar{u})$ is the boundary in the asymptotically flat coordinates (3.11); at $\bar{u}<\bar{u}_{e}$ and $\bar{u}>\bar{u}_{e}$ it represents $U(v)$ and $U_{2}(v)$, respectively.

If the state of the outgoing radiation was pure at $-\infty<\bar{u}<+\infty$, the geometric entropies $\Sigma^{-}$and $\Sigma^{+}$would coincide because their intervals are complementary. ${ }^{11}$ This equality is automatic in eq. (4.3) for any smooth $\bar{v}(\bar{u})$. Physically, $\Sigma^{+}=\Sigma^{-}$would mean that the Hawking radiation is entangled with the remnant [28] - a state of quantum fields beyond the endpoint. However our boundary has an unavoidable jump at $\bar{u}=\bar{u}_{e}$, and we are going to demonstrate that it makes the two entropies essentially different.

Start with $\Sigma^{-}$for the Hawking radiation. To make the estimate transparent, we assume that the incoming matter is well localized, has large energy, and therefore collides with the boundary after crossing the horizon in figure $2(\mathrm{c})$. Then $U(v)$ is approximately given by eq. (3.22), and we obtain,

$$
\Sigma^{-} \approx 4 \pi Q^{2} \log \left[-v_{\times} \partial_{v} U\left(v_{\times}\right) / u_{e}\right] \approx 4 \pi Q^{2} \log \frac{v_{e}}{v_{\times}} \approx \frac{4 \pi}{\lambda}\left(E_{\mathrm{in}}-M_{c r}\right),
$$

where we introduced the crossing time $U\left(v_{\times}\right)=0$, applied eq. (3.29), and ignored the terms suppressed by $Q^{2}$. Expression (4.4) coincides with the thermal entropy of one-dimensional gas with energy $E_{\text {in }}-M_{c r}$ and temperature $T_{H}=\lambda /(2 \pi)$. Thus, the entanglement entropy of the Hawking radiation has the maximal possible value. It can be arbitrarily large at high $E_{\text {in }}$. This reproduces the standard result.

The "remnant" entropy $\Sigma^{+}$in eq. (4.3) depends on the apparent initial velocity $\partial_{v} U_{2}\left(v_{e}\right)$ of the second boundary, which is not fixed. In section 3.4 we demonstrated,

\footnotetext{
${ }^{11}$ For a time, we ignore the entropy of the thunderpop at $\bar{u}=\bar{u}_{e}$.
} 
however, that this velocity satisfies the inequality (3.30), or the spacetime would be singular beyond the endpoint. We thus obtain,

$$
\Sigma^{+}=4 \pi Q^{2} \log \left[-v_{e} \partial_{v} U_{2}\left(v_{e}\right) / u_{e}\right]<4 \pi Q^{2} \log \left[-Q^{2} /\left(4 \lambda^{2} u_{e} v_{e}\right)\right] \sim O\left(Q^{2}\right),
$$

where eq. (3.29) was used in the last equality. Thus, non-thermal radiation beyond the endpoint is almost pure. Parametric difference between eqs. (4.4) and (4.5) is the essence of the Hawking information paradox.

One can try to store the entanglement entropy into the thunderpop which emanates from the endpoint singularity and can be in any quantum state. For example, introducing tanh-like smoothing of $\bar{v}(\bar{u})$ at $\bar{u}=\bar{u}_{e}$, one automatically obtains $\Sigma^{-}=\Sigma^{+}$in eq. (4.3). The entropies remain equal even in the limit when the smoothing region becomes small, $\delta \bar{u} \rightarrow 0$. However, the same smoothing introduces an infinite-energy thunderbolt going along the last ray. Indeed, the Schwarzian reflection law (3.13) gives,

$$
\int_{\bar{u}_{e}-\delta \bar{u}}^{\bar{u}_{e}+\delta \bar{u}} d \bar{u} \bar{T}_{\bar{u} \bar{u}} \sim Q^{2} \int_{\bar{u}_{e}-\delta \bar{u}}^{\bar{u}_{e}+\delta \bar{u}} d \bar{u}\left(\frac{\partial_{\bar{u}}^{2} \bar{v}}{\partial_{\bar{u}} \bar{v}}\right)^{2} \geq \frac{Q^{2}}{2 \delta \bar{u}}\left(\int_{\bar{u}_{e}-\delta \bar{u}}^{\bar{u}_{e}+\delta \bar{u}} \frac{\partial_{\bar{u}}^{2} \bar{v}}{\partial_{\bar{u}} \bar{v}}\right)^{2}=\left.\frac{\left(\Sigma^{-}-\Sigma^{+}\right)^{2}}{32 \pi^{2} \delta \bar{u} Q^{2}}\right|_{\text {unreg }},
$$

where we ignored all the terms regular at $\delta \bar{u} \rightarrow 0$, used the Cauchy inequality, and expressed the result via the entropy mismatch between eqs. (4.4) and (4.5). We see that the energy of the "thunderbolt" diverges at $\delta \bar{u} \rightarrow 0$. Moreover, "Planckian" thunderbolt with $\delta \bar{u} \sim \lambda^{-1}$ has energy $E_{\mathrm{in}}^{2} /\left(\lambda Q^{2}\right)$ which parametrically exceeds the black hole mass. Thus, our small innocent thunderpop cannot recover the information. To do that, radical large-distance modification of the semiclassical geometry is needed.

There is another curious property of the RST black holes which is apparent in our calculations. Recall that the black hole mass reaches a finite critical value $M \approx M_{c r}=2 \lambda \mathrm{e}^{-2 \phi_{0}}$ at the endpoint of evaporation. Nevertheless, this critical black hole decays into an almost pure state with small entanglement entropy, see eq. (4.5). Thus, the thermodynamical entropy $S_{\mathrm{BH}}$ of this object should be also small. Direct calculations of the black hole entropy $[57,58,60,61]$ are consistent with this unusual property. They give expression,

$$
S_{\mathrm{BH}}=4 \pi\left(\hat{\phi}_{\mathrm{hor}}-\hat{\phi}_{0}\right)
$$

which reaches zero at the endpoint when the value $\hat{\phi}_{\text {hor }}$ of the JT dilaton at the apparent horizon equals $\hat{\phi}_{0}$. However, while the mass-dependent part of Eq. (4.6) is fixed by the black hole thermodynamics, the constant part is added somewhat ad hoc, using additional physical considerations. Our results independently confirm that $S_{\mathrm{BH}}=0$ at $\phi_{\text {hor }}=\phi_{0} . \mathrm{We}$ are going to further address this question in the forthcoming publication [59].

\subsection{Non-conservation of a global charge}

It has long been believed that quantum gravity does not tolerate any conserved global charges. Indeed, by causality black holes evaporate into all sorts of particles regardless to what they were made of, violating all global quantum numbers. 
The RST action (2.1), (2.11) has a global shift symmetry $f \rightarrow f+$ const and the respective conserved current ${ }^{12} j^{\mu}=\nabla^{\mu} f$. This gives the asymptotic conservation law,

$$
0=\int d^{2} x \sqrt{-g} \nabla_{\mu} j^{\mu}=\int_{\phi=\phi_{0}} d \tau n^{\mu} \nabla_{\mu} f-\int d \bar{v} \partial_{\bar{v}} f_{\text {in }}(\bar{v})+\int d \bar{u} \partial_{\bar{u}} f_{\text {out }}(\bar{u})
$$

where we used the Gauss theorem with boundary terms coming from the line $\phi=\phi_{0}$ and two light-like infinities. Recall that $n^{\mu}$ and $\tau$ are the outer normal and proper time of the boundary, whereas $(\bar{u}, \bar{v})$ represent asymptotically flat coordinates. If the spacetime has simple topology like in figure 2(a), the matter fields satisfy Neumann conditions at the boundary, and the conservation law

$$
\mathcal{Q} \equiv f_{\text {in }}(\bar{v}=+\infty)-f_{\text {in }}(\bar{v}=-\infty)=f_{\text {out }}(\bar{u}=+\infty)-f_{\text {out }}(\bar{u}=-\infty)
$$

holds. But once the black hole appears in figure 2(c), this law gets broken because $n^{\mu} \nabla_{\mu} f \neq 0$ at the spatial sections of the line $\phi=\phi_{0}$.

Figure 5(b) illustrates violation of the charge (4.7) by the evaporating black hole. In this figure the incoming wave packet $f_{\text {in }}(\bar{v})$ approaches $f^{\prime}$ at $\bar{v} \rightarrow \pm \infty$ taking the value $f^{\prime \prime}$ in between (grey region). Its global charge is zero. But reflection from the boundary gives $f_{\text {out }}=f^{\prime \prime}$ at the endpoint $S^{\prime}$ of black hole evaporation, and by continuity ${ }^{13}$ — in the entire region beyond the last ray $S^{\prime} L$. Then the global charge of $f_{\text {out }}$ is $\mathcal{Q}_{\text {out }} \equiv f^{\prime \prime}-f^{\prime} \neq 0$.

One can again try to pack the compensating charge inside the thunderpop, to save the conservation law. But this requires energy, like in the case with entropy. The energy of the thunderpop in asymptotic coordinates is

$$
E_{\text {th-pop }}=\int_{\bar{u}_{e}-\delta \bar{u}}^{\bar{u}_{e}+\delta \bar{u}} d \bar{u}\left(\partial_{\bar{u}} f_{\text {out }}\right)^{2} \geq \frac{1}{2 \delta u}\left(\int_{\bar{u}_{e}-\delta \bar{u}}^{\bar{u}_{e}+\delta \bar{u}} d \bar{u} \partial_{\bar{u}} f_{\text {out }}\right)^{2}=\frac{\mathcal{Q}_{\text {th-pop }}^{2}}{2 \delta \bar{u}}
$$

where $\delta \bar{u}$ is the size of the uncontrollable region, we used the Cauchy inequality and denoted $\mathcal{Q}_{\text {th-pop }}=f_{\text {out }}\left(\bar{u}_{e}+\delta \bar{u}\right)-f_{\text {out }}\left(\bar{u}_{e}-\delta \bar{u}\right)$. On the other hand, the incoming energy is $E_{\text {in }} \sim\left(f^{\prime \prime}-f^{\prime}\right)^{2} / \Delta \bar{v}=\mathcal{Q}_{\text {out }}^{2} / \Delta \bar{v}$, where $\Delta \bar{v}$ is a typical width of the incoming energy flux. Thus, $\mathcal{Q}_{\text {th-pop }} \ll \mathcal{Q}_{\text {out }}$, if we want to keep $E_{\text {th-pop }}<E_{\text {in }}$ and $\delta \bar{u} \ll \Delta \bar{v}$. The other (unphysical) options would be to introduce a large naked singularity with $\delta \bar{u} \sim \Delta \bar{v}$ or give the thunderpop energy exceeding $E_{\text {in }}$.

We conclude that charge non-conservation in our model is robust against quantum corrections at the last ray.

\section{Discussion}

In this paper we demonstrated local equivalence between the quantum RST and flat-space JT models, and applied it to describe evaporating black holes. We regularized the singularities of the RST fields with the reflective boundary and derived one-loop effective action

\footnotetext{
${ }^{12}$ Actually, $N$ currents - one per matter field.

${ }^{13}$ This does not contradict to the reflection law of $f$ which has an integration constant: $f_{\text {out }}(U(v))=f_{\text {in }}(v)+$ const, cf. eq. (3.12).
} 
satisfying all self-consistency requirements. Still, our semiclassical black hole solutions are not satisfactory in three respects. First, they violate the boundary conditions at the spacelike line $\phi=\phi_{0}$ behind the horizon and therefore do not extremize the effective action. This deprives the solutions from their original role of saddle-point configurations for the path integral and therefore makes all further physical interpretations speculative. Second, the endpoint of evaporation corresponds to a naked singularity with divergent action. Regularizing the action, one introduces arbitrary counter-terms which determine the subsequent evolution. Third and as a consequence of the first two, the final state of Hawking radiation has large entanglement entropy indicating unitarity loss.

One can search for the root of the above problems in two directions. The first suspect is the semiclassical method. There are many situations in semiclassical physics where the "naive" solutions do not exist: they are either singular [63] or do not satisfy necessary boundary conditions [64], just like the solutions in the RST model. In this case one can apply the method of constrained instantons [63], i.e. enforce correct behavior of the solutions with additional constraint and then integrate over the constraint in the path integral. This procedure was proposed for the black hole evaporation [62], but has never been used beyond the simplest thin shell models.

Also, one can make the semiclassical method work by computing a different quantity. For example, consider the unitarity relation,

$$
\mathrm{e}^{\int d k a_{k}^{\dagger} b_{k}}=\left\langle a\left|\hat{\mathcal{S}}^{\dagger} \hat{\mathcal{S}}\right| b\right\rangle=\int\left[d c^{\dagger} d c\right] \mathrm{e}^{-\int d k c_{k}^{\dagger} c_{k}}\langle c|\hat{\mathcal{S}}| a\rangle^{\dagger}\langle c|\hat{\mathcal{S}}| b\rangle,
$$

where $|a\rangle,|b\rangle$, and $|c\rangle$ are the coherent Fock states of matter fields in flat spacetime with amplitudes $a_{k}, b_{k}$, and $c_{k}$, while $\hat{\mathcal{S}}$ is the $\mathcal{S}$-matrix of the RST model. At $a \neq b$ eq. (5.1) involves exponentially suppressed coherent amplitudes of black hole formation and decay into a prescribed final state. The processes of this kind are described by complex semiclassical solutions with distinct properties, cf. [65] and [62, 66]. Using ${ }^{14}$ the latter in eq. (5.1), one can directly test unitarity of the RST $\mathcal{S}$-matrix.

As a second possibility, one notes that the "regularization" boundary may ruin unitarity of the flat-space JT model. Indeed, the boundary action (2.11) describes point particle stiffly coupled to the dilaton field, with trajectory following the line $\phi=\phi_{0}$. However, models with first-quantized relativistic particles are generically non-unitary due to Klein paradox [67]: computation of transition probabilities in these models gives unphysical results at energies exceeding the threshold for particle-antiparticle production. Our RST solutions demonstrate similar behavior. To remedy this inconsistency, one can try to second-quantize the boundary, promoting it to a quantum field. This may restore unitarity due to boundary-antiboundary production.

\section{Acknowledgments}

We are grateful to Dmitry Eremeev for collaboration at early stages of this project. We also thank Sergey Sibiryakov, and Sergei Dubovsky for fruitful discussions. D.L. thanks

\footnotetext{
${ }^{14}$ The integral over $c_{k}$ and $c_{k}^{\dagger}$ can be evaluated in the saddle-point approximation.
} 
Université libre de Bruxelles for hospitality. This work was supported by the grant RSF 16-12-10494.

\section{A Deriving the semiclassical equations}

\section{A.1 Solution in the bulk}

We obtain the semiclassical field equations by extremizing the effective action (3.1) with respect to the background fields. In particular, the matter fields $f=f_{1}$ and $\chi$ satisfy,

$$
\square f=0, \quad \square \chi+Q R=0,
$$

while variation with respect to $g^{\mu \nu}$ gives,

$$
2\left(2 \mathrm{e}^{-2 \phi}+Q^{2}\right)\left(\nabla_{\mu} \nabla_{\nu}-g_{\mu \nu} \square\right) \phi+4 g_{\mu \nu} \mathrm{e}^{-2 \phi}\left[(\nabla \phi)^{2}-\lambda^{2}\right]=\mathcal{T}_{\mu \nu}^{(f)}+\mathcal{T}_{\mu \nu}^{(\chi)} .
$$

In the last equation we introduced the energy-momentum tensors,

$$
\begin{aligned}
& \mathcal{T}_{\mu \nu}^{(f)}=\nabla_{\mu} f \nabla_{\nu} f-\frac{1}{2} g_{\mu \nu}(\nabla f)^{2}, \\
& \mathcal{T}_{\mu \nu}^{(\chi)}=\nabla_{\mu} \chi \nabla_{\nu} \chi-\frac{1}{2} g_{\mu \nu}(\nabla \chi)^{2}+2 Q\left(\nabla_{\mu} \nabla_{\nu}-g_{\mu \nu} \square\right) \chi
\end{aligned}
$$

which are conserved due to eqs. (A.1): $\nabla^{\mu} \mathcal{T}_{\mu \nu}^{(f)}=\nabla^{\mu} \mathcal{T}_{\mu \nu}^{(\chi)}=0$. Semiclassical equation for $\phi$ can be written in the form

$$
\square \phi+R / 2=0
$$

using eqs. (A.1) and (A.2).

Note that the RST vacuum (3.10) satisfies eqs. (A.1), (A.2), (A.5) because in flat RST coordinates (3.11) it gives $\partial_{\mu} \partial_{\nu} \phi=0,\left(\partial_{\mu} \phi\right)^{2}=\lambda^{2}, R=0$, and $\chi=0$ due to eq. (3.2).

In the conformal gauge (2.2) eqs. (A.1) and (A.5) simplify,

$$
\partial_{u} \partial_{v} f=0, \quad \partial_{u} \partial_{v}(\chi-2 Q \rho)=0, \quad \partial_{u} \partial_{v}(\phi-\rho)=0,
$$

where we substituted $R=8 \mathrm{e}^{-2 \rho} \partial_{u} \partial_{v} \rho$. Solutions of the first two equations are given in eq. (3.2), where the in- and outgoing wave packets $f_{\text {in }}(v), \chi_{\text {in }}(v)$ and $f_{\text {out }}(u), \chi_{\text {out }}(u)$ are arbitrary. The last equation together with the residual reparametrization invariance $u \rightarrow \tilde{u}(u), v \rightarrow \tilde{v}(v)$ allows us to impose the "Kruskal" gauge $\rho=\phi$ in eq. (3.6).

Substituting the matter fields (3.2) into eqs. (A.3), (A.4), one computes their energymomentum tensors,

$$
\mathcal{T}_{u u}^{(f)}+\mathcal{T}_{u u}^{(\chi)}=4 Q^{2}\left[\partial_{u}^{2} \rho-\left(\partial_{u} \rho\right)^{2}\right]+T_{u u}, \quad \mathcal{T}_{u v}^{(f)}+\mathcal{T}_{u v}^{(\chi)}=-4 Q^{2} \partial_{u} \partial_{v} \rho,
$$

where expression for the $(v v)$ component can be obtained by replacing $u \rightarrow v$. In eq. (A.7) we separated the contributions $T_{u u}(u)$ and $T_{v v}(v)$ of the incoming and outgoing matter wave packets defined in eq. (3.4). By themselves, $T_{v v}$ and $T_{u u}$ are not tensors. However, they coincide with the energy fluxes at infinity in the asymptotically flat $(\rho=0)$ coordinates $\bar{u}$ and $\bar{v}$. 
Using eqs. (A.7) in the "Kruskal" gauge $\rho=\phi$, we rewrite eq. (A.2) as

$$
\partial_{u}^{2} \hat{\phi}=-T_{u u} / 2, \quad \partial_{v}^{2} \hat{\phi}=-T_{v v} / 2, \quad \partial_{u} \partial_{v} \hat{\phi}=-\lambda^{2} .
$$

Recall that $\hat{\phi}=\mathrm{e}^{-2 \phi}+Q^{2} \phi$ is the JT field introduced in eq. (1.1). General solution of eq. (A.8) is

$$
\hat{\phi}=-\lambda^{2} u v+c_{1}+c_{2} u+c_{3} v+g(v)+h(u),
$$

where the primitives $g$ and $h$ are given by eqs. (3.8), (3.9).

In the main text we consider solutions starting from the RST vacuum (3.10) in the asymptotic past. This means that the spacetime is flat, $R=0$, and $\phi=-\lambda \bar{x}$ in the beginning, see eq. (3.11). On the other hand, the coordinates $u$ and $v$ cover semi-infinite intervals in figure 1(b). We can shift them to the domains $-\infty<u<0$ and $0<v<+\infty$. Then the past time infinity $i^{-}$is reached at $u \rightarrow-\infty, v \rightarrow 0$, and the dilaton field $\hat{\phi} \rightarrow-\lambda^{2} u v-Q^{2} \log \left(-\lambda^{2} u v\right) / 2+c_{1}+c_{2} u$ should be a finite function of $\bar{x}$ in this limit, see eq. (A.9). Thus, $c_{2}=0$ and $u v$ is finite at $i^{-}$. Moreover, we compute the curvature plugging the past asymptotics of $\hat{\phi}$ into eq. (A.5) and find,

$$
R \rightarrow-\mathrm{e}^{-2 \phi}\left(\mathrm{e}^{-2 \phi}+\lambda^{2} u v\right) \frac{\left(Q^{4}+4 \lambda^{2} u v \mathrm{e}^{-2 \phi}\right)}{u v\left(Q^{2} / 2-\mathrm{e}^{-2 \phi}\right)^{3}} \quad \text { as } \quad u \rightarrow-\infty .
$$

Thus, in the regime (2.12) the spacetime is flat in the past only if $\mathrm{e}^{-2 \phi}=-\lambda^{2} u v$. This gives $c_{1}=0$ in eq. (A.9). Note finally that the future time infinity $i^{+}$is reached at $v \rightarrow+\infty$, $u \rightarrow 0$ and finite $\hat{\phi}$. This is possible only if $c_{3}=0$ in eq. (A.9). At $c_{1}=c_{2}=c_{3}=0$ the solution (A.9) reduces to eq. (3.7) from the main text.

\section{A.2 Reflection laws}

We obtain boundary conditions by varying the effective action (3.1) with respect to the boundary values of all fields. Due to reparametrization invariance we can consider only particular variations preserving the coordinate position of the boundary: $\delta \phi=0$ there. Then [38, 47, 49]

$$
\begin{aligned}
\delta S=\int d \tau\left\{h^{\mu \nu} \delta h_{\mu \nu}\left[\nabla_{n}\left(\mathrm{e}^{-2 \phi}-Q^{2} \phi+Q \chi\right)-\mu / 2\right]\right. & \\
& \left.-\delta \chi\left(\nabla_{n} \chi-2 K Q\right)-\delta f \nabla_{n} f\right\}
\end{aligned}
$$

where we left only the boundary terms, introduced the outer normal derivative $\nabla_{n} \equiv n^{\mu} \nabla_{\mu}$ and the induced metric $h_{\mu \nu} \equiv g_{\mu \nu}-n_{\mu} n_{\nu}$. As before, we keep only one matter field $f=f_{1}$. Then the semiclassical boundary conditions are,

$$
\nabla_{n} \chi=2 Q K, \quad \nabla_{n} f=0, \quad\left(2 \mathrm{e}^{-2 \phi_{0}}+Q^{2}\right)\left(\nabla_{n} \phi-\lambda\right)=2 Q^{2} K,
$$

where the value of $\mu$ was taken from section 2.2 .

Note that the flat RST vacuum (3.10), (3.11) with the static boundary (3.15) satisfies eqs. (A.12): in this case $K=0, \chi=0$ and $\nabla_{n} \phi=\lambda$. 
In "Kruskal" coordinates $(u, v)$ with $\rho=\phi$ the boundary is described by the function $u=U(v)$. The outer normal has components

$$
\left\{n^{u}, n^{v}\right\}=\mathrm{e}^{-\phi}\left\{\left(\partial_{v} U\right)^{1 / 2},-\left(\partial_{v} U\right)^{-1 / 2}\right\},
$$

where $\partial_{v} U>0$ for the timelike boundary. Substituting the extrinsic curvature $K=\nabla_{\mu} n^{\mu}=n^{\nu} \partial_{\nu} \phi-\mathrm{e}^{-\phi} \partial_{v}\left(\partial_{v} U\right)^{-1 / 2}$ into the first two of eqs. (A.12), we obtain the boundary conditions

$$
\partial_{v} U \partial_{u} f_{\text {out }}=\partial_{v} f_{\text {in }}, \quad \partial_{v} U \partial_{u} \chi_{\text {out }}=\partial_{v} \chi_{\text {in }}+Q \partial_{v} \log \left(\partial_{v} U\right) \quad \text { at } \quad u=U(v) .
$$

Solutions of these equations are the reflection laws of matter fields (3.12) and of their energy fluxes (3.13).

The latter reflection laws together with the bulk constraint give equation for $U(v)$. Indeed, full derivative of $\hat{\phi}(U(v), v)$ along the boundary is zero, hence,

$$
\partial_{u} h-\lambda^{2} v=-\left(\partial_{v} g-\lambda^{2} U\right) / \partial_{v} U \quad \text { at } \quad u=U(v),
$$

where eq. (3.7) was used. We take derivative of eq. (A.14) along the boundary and then express $\partial_{u}^{2} h$ from eqs. (3.9), (3.13). We find,

$$
\partial_{v}\left(\frac{\partial_{v} g-\lambda^{2} U}{\sqrt{\partial_{v} U}}\right)=\frac{Q^{2}}{2} \partial_{v}\left(\frac{\partial_{v}^{2} U}{\left(\partial_{v} U\right)^{3 / 2}}\right)
$$

Integrating this equation, one arrives at eq. (3.14) with arbitrary constant in front of the right-hand side.

The last boundary condition in eqs. (A.12) fixes the value of the multiplicative constant in eq. (3.14). Indeed, we have already shown that the RST vacuum (3.10), (3.15) satisfies the entire set of semiclassical equations and boundary conditions. On the other hand, $U(v)$ in eq. (3.15) agrees with eq. (3.14) only if the constant in that equation equals $\mathrm{e}^{2 \phi_{0}} q^{2} / \lambda^{2}$. One can explicitly check that once this value is fixed, the third of eqs. (A.12) is equivalent to eq. (3.14).

\section{A.3 Energy conservation}

We introduce the energies $E_{\text {in }}$ and $E_{\text {out }}$ of the incoming and outgoing matter by recalling that the fluxes $\bar{T}_{\bar{u} \bar{u}}$ and $\bar{T}_{\bar{v} \bar{v}}$ coincide with the respective components of the energymomentum tensor $\mathcal{T}_{\mu \nu}$ in the asymptotically flat coordinates $(\bar{u}, \bar{v})$ with $\rho=0$, see eq. (A.7). Thus,

$$
E_{\text {in }}=\int_{-\infty}^{+\infty} d \bar{v} \bar{T}_{\bar{v} \bar{v}}(\bar{v}), \quad \quad E_{\text {out }}=\int_{-\infty}^{+\infty} d \bar{u} \bar{T}_{\bar{u} \bar{u}}(\bar{u})
$$

Transforming to the "Kruskal" frame by eqs. (3.5), (3.11), one gets,

$$
E_{\text {in }}=-2 \lambda \int_{0}^{+\infty} d v v\left(\partial_{v}^{2} g-\frac{Q^{2}}{2 v^{2}}\right), \quad E_{\text {out }}=2 \lambda \int_{-\infty}^{0} d u u\left(\partial_{u}^{2} h-\frac{Q^{2}}{2 u^{2}}\right),
$$

where $g(v)$ and $h(u)$ are the second primitives of $T_{v v}$ and $T_{u u}$ in eqs. (3.8), (3.9). 
Let us demonstrate that the energy is conserved, $E_{\text {in }}=E_{\text {out }}$, for the semiclassical solutions starting from the RST vacuum in the past and arriving to it in the asymptotic future. A black hole in the intermediate state, if it evaporates completely, does not affect this conservation law.

We introduce the integration limits $v_{1} \rightarrow 0, v_{2} \rightarrow+\infty, u_{1} \rightarrow-\infty$, and $u_{2} \rightarrow 0$ in eq. (A.17). Since the trajectory of the boundary approaches the vacuum solution (3.15) at $v \rightarrow 0$ and $v \rightarrow+\infty$, we choose $u_{1}=U\left(v_{1}\right)$ and $u_{2}=U\left(v_{2}\right)$. Performing the integrals in eq. (A.17), one obtains [47],

$$
\frac{E_{\mathrm{in}}-E_{\mathrm{out}}}{2 \lambda}=\left.\left\{g(v)+h(U(v))+\frac{Q^{2}}{2} \log \left(-\lambda^{2} v U(v)\right)-v \partial_{v} g-U \partial_{u} h\right\}\right|_{v_{1}} ^{v_{2}}=0
$$

where in the last equality we used eqs. (3.15), (3.7), recalled that $\phi=\phi_{0}$ along the boundary, and evaluated the limits $v_{1} \rightarrow 0, v_{2} \rightarrow+\infty$. This proves energy conservation.

\section{A.4 Equations for solvable deformation}

Consider the model [47] with Dirichlet boundary condition (3.19) for the quantum field $\chi$. Boundary variation of the metric in eq. (A.11) gives,

$$
n^{\mu} \nabla_{\mu}\left(e^{-2 \phi}-Q^{2} \phi+Q \chi\right)=-\lambda\left(2 e^{-2 \phi_{0}}+Q^{2}\right) .
$$

Using the outer normal (A.13), dilaton (3.7), eq. (A.14), and reflection law for $\chi$ in the gauge $\rho=\phi$, we obtain equation,

$$
\partial_{v} U=e^{2 \phi_{0}} \frac{q^{2}}{\lambda^{2}}\left(\partial_{v} g+Q \partial_{v} \chi_{\mathrm{in}}-\lambda^{2} U\right)^{2} .
$$

We take the incoming quantum field in vacuum, $\chi_{\text {in }}=Q \log (\lambda v)$, and arrive to eq. (3.21) from the main text.

\section{B Entanglement entropy}

In this appendix we review the geometric entropy $\Sigma\left(\bar{v}_{1}, \bar{v}_{2}\right)$ measuring entanglement of the quantum field inside the interval $\left[\bar{v}_{1}, \bar{v}_{2}\right]$ with the rest of the world. In the particular case of one massless scalar field in the vacuum state this entropy equals [40],

$$
\Sigma_{\mathrm{vac}}\left(\bar{v}_{1}, \bar{v}_{2}\right)=\frac{1}{12} \log \left[\frac{\left(\bar{v}_{2}-\bar{v}_{1}\right)^{2}}{\delta \bar{v}_{1} \delta \bar{v}_{2}}\right]
$$

where we restricted attention to the left-moving sector and introduced UV cutoffs $\delta \bar{v}_{1}, \delta \bar{v}_{2}$ at the borders of the interval. In quantum theory (B.1) is a divergent quantity which should be renormalized. In thermodynamics, $\delta \bar{v}_{1}$ and $\delta \bar{v}_{2}$ are the parameters of coarse-graining.

Importantly, the entanglement entropy is conformally invariant [40]. Indeed, general conformal transformation is local. It may distort the state of the quantum field but never changes the degrees of freedom inside the interval. Selecting one RST field - say, $f_{N}-$ 
one recalls that it is initially in vacuum and its reflection from the boundary is a conformal transformation (3.12). Thus, the outgoing entropy of this field is,

$$
\Sigma\left(\bar{u}_{1}, \bar{u}_{2}\right)=\Sigma_{\mathrm{vac}}\left(\bar{v}\left(\bar{u}_{1}\right), \bar{v}\left(\bar{u}_{2}\right)\right)=\frac{1}{12} \log \left[\frac{\left(\bar{v}\left(\bar{u}_{2}\right)-\bar{v}\left(\bar{u}_{1}\right)\right)^{2}}{\delta \bar{u}_{1} \delta \bar{u}_{2} \bar{v}^{\prime}\left(\bar{u}_{1}\right) \bar{v}^{\prime}\left(\bar{u}_{2}\right)}\right]
$$

where we introduced the trajectory $\bar{v}=\bar{v}(\bar{u})$ of the boundary in asymptotic coordinates and related the cutoffs $\delta \bar{v}\left(\bar{u}_{i}\right)$ to $\delta \bar{u}_{i}$; primes are the $\bar{u}$-derivatives. We stress that the outgoing field $f_{N \text {, out }}$ with entropy (B.2) is not in vacuum.

Finally, we regularize the entropy (B.2) by subtracting its vacuum value,

$$
\Sigma_{\mathrm{reg}}\left(\bar{u}_{1}, \bar{u}_{2}\right)=\Sigma\left(\bar{u}_{1}, \bar{u}_{2}\right)-\Sigma_{\mathrm{vac}}\left(\bar{u}_{1}, \bar{u}_{2}\right)=\frac{1}{12} \log \left[\frac{\left(\bar{v}\left(\bar{u}_{2}\right)-\bar{v}\left(\bar{u}_{1}\right)\right)^{2}}{\left(\bar{u}_{2}-\bar{u}_{1}\right)^{2} \bar{v}^{\prime}\left(\bar{u}_{2}\right) \bar{v}^{\prime}\left(\bar{u}_{1}\right)}\right] .
$$

This quantity measures entanglement of the outgoing field within the interval $\left[\bar{u}_{1}, \bar{u}_{2}\right]$ relative to the vacuum. In the main text we use half-infinite intervals $(-\infty, \bar{u})$ and $(\bar{u},+\infty)$. Sending $\bar{u}_{2} \rightarrow \pm \infty$ and recalling that in this limit $\bar{v}\left(\bar{u}_{2}\right) \rightarrow \bar{u}_{2}-2 \phi_{0} / \lambda$, we obtain

$$
\Sigma_{\mathrm{reg}}(-\infty, \bar{u})=\Sigma_{\mathrm{reg}}(\bar{u},+\infty)=-\frac{1}{12} \log \bar{v}^{\prime}(\bar{u}) .
$$

Multiplication by the number of fields $N=48 \pi Q^{2}$ gives eq. (4.3).

Open Access. This article is distributed under the terms of the Creative Commons Attribution License (CC-BY 4.0), which permits any use, distribution and reproduction in any medium, provided the original author(s) and source are credited.

\section{References}

[1] C. Teitelboim, Gravitation and Hamiltonian structure in two space-time dimensions, Phys. Lett. B 126 (1983) 41 [INSPIRE].

[2] R. Jackiw, Lower Dimensional Gravity, Nucl. Phys. B 252 (1985) 343 [InSPIRE].

[3] D. Cangemi and R. Jackiw, Gauge invariant formulations of lineal gravity, Phys. Rev. Lett. 69 (1992) 233 [hep-th/9203056] [INSPIRE].

[4] S. Dubovsky, V. Gorbenko and M. Mirbabayi, Asymptotic fragility, near AdS $S_{2}$ holography and TT, JHEP 09 (2017) 136 [arXiv: 1706.06604] [inSPIRE].

[5] S. Dubovsky, V. Gorbenko and G. Hernández-Chifflet, $T \bar{T}$ partition function from topological gravity, JHEP 09 (2018) 158 [arXiv:1805.07386] [INSPIRE].

[6] P. Saad, S.H. Shenker and D. Stanford, JT gravity as a matrix integral, arXiv:1903.11115 [INSPIRE].

[7] D. Stanford and E. Witten, JT gravity and the ensembles of random matrix theory, arXiv: 1907.03363 [INSPIRE].

[8] S.W. Hawking, Particle creation by black holes, Commun. Math. Phys. 43 (1975) 199 [Erratum ibid. 46 (1976) 206] [INSPIRE].

[9] S.W. Hawking, Breakdown of predictability in gravitational collapse, Phys. Rev. D 14 (1976) 2460 [INSPIRE]. 
[10] J.M. Maldacena, Eternal black holes in Anti-de Sitter, JHEP 04 (2003) 021 [hep-th/0106112] [INSPIRE].

[11] G. Penington, Entanglement wedge reconstruction and the information paradox, arXiv: 1905.08255 [INSPIRE].

[12] A. Almheiri, N. Engelhardt, D. Marolf and H. Maxfield, The entropy of bulk quantum fields and the entanglement wedge of an evaporating black hole, JHEP 12 (2019) 063 [arXiv: 1905.08762] [INSPIRE].

[13] G. Penington, S.H. Shenker, D. Stanford and Z. Yang, Replica wormholes and the black hole interior, arXiv: 1911.11977 [INSPIRE].

[14] F.F. Gautason, L. Schneiderbauer, W. Sybesma and L. Thorlacius, Page curve for an evaporating black hole, JHEP 05 (2020) 091 [arXiv:2004.00598] [INSPIRE].

[15] A. Almheiri, D. Marolf, J. Polchinski and J. Sully, Black holes: complementarity or firewalls?, JHEP 02 (2013) 062 [arXiv: 1207.3123] [INSPIRE].

[16] Y. Zeldovich, A new type of radioactive decay: gravitational annihilation of baryons, Phys. Lett. A 59 (1976) 254 [INSPIRE].

[17] S.R. Coleman and S. Hughes, Black holes, wormholes and the disappearance of global charge, Phys. Lett. B 309 (1993) 246 [hep-th/9305123] [INSPIRE].

[18] D. Stojkovic, F.C. Adams and G.D. Starkman, Information-preserving black holes still do not preserve baryon number and other effective global quantum numbers, Int. J. Mod. Phys. D 14 (2005) 2293 [gr-qc/0604072] [INSPIRE].

[19] J.G. Russo, L. Susskind and L. Thorlacius, The endpoint of Hawking radiation, Phys. Rev. D 46 (1992) 3444 [hep-th/9206070] [INSPIRE].

[20] C.G. Callan Jr., S.B. Giddings, J.A. Harvey and A. Strominger, Evanescent black holes, Phys. Rev. D 45 (1992) 1005 [hep-th/9111056] [inSPIRE].

[21] A. Strominger, Faddeev-Popov ghosts and $(1+1)$-dimensional black hole evaporation, Phys. Rev. D 46 (1992) 4396 [hep-th/9205028] [InSPIRE].

[22] S. Dubovsky, private communication.

[23] T. Banks, A. Dabholkar, M.R. Douglas and M. O'Loughlin, Are horned particles the climax of Hawking evaporation?, Phys. Rev. D 45 (1992) 3607 [hep-th/9201061] [INSPIRE].

[24] J.G. Russo, L. Susskind and L. Thorlacius, Black hole evaporation in $(1+1)$-dimensions, Phys. Lett. B 292 (1992) 13 [hep-th/9201074] [INSPIRE].

[25] L. Thorlacius, Black hole evolution, Nucl. Phys. B Proc. Suppl. 41 (1995) 245 [hep-th/9411020] [INSPIRE].

[26] S.P. de Alwis, Quantization of a theory of 2D dilaton gravity, Phys. Lett. B 289 (1992) 278 [hep-th/9205069] [INSPIRE].

[27] J. Brown, M. Henneaux and C. Teitelboim, Black holes in two space-time dimensions, Phys. Rev. D 33 (1986) 319 [INSPIRE].

[28] A. Almheiri and J. Sully, An uneventful horizon in two dimensions, JHEP 02 (2014) 108 [arXiv: 1307.8149] [INSPIRE].

[29] J.G. Russo, L. Susskind and L. Thorlacius, Cosmic censorship in two-dimensional gravity, Phys. Rev. D 47 (1993) 533 [hep-th/9209012] [INSPIRE]. 
[30] T.D. Chung and H.L. Verlinde, Dynamical moving mirrors and black holes, Nucl. Phys. B 418 (1994) 305 [hep-th/9311007] [INSPIRE].

[31] A.M. Polyakov, Quantum geometry of bosonic strings, Phys. Lett. B 103 (1981) 207 [INSPIRE].

[32] J. Polchinski, String theory. Volume 1: an introduction to the bosonic string, Cambridge University Press, Cambridge U.K. (1998).

[33] A. Strominger and L. Thorlacius, Conformally invariant boundary conditions for dilaton gravity, Phys. Rev. D 50 (1994) 5177 [hep-th/9405084] [INSPIRE].

[34] S.R. Das and S. Mukherji, Boundary dynamics in dilaton gravity, Mod. Phys. Lett. A 9 (1994) 3105 [hep-th/9407015] [INSPIRE].

[35] E.P. Verlinde and H.L. Verlinde, $A$ unitary $S$ matrix and $2 D$ black hole formation and evaporation, Nucl. Phys. B 406 (1993) 43 [hep-th/9302022] [INSPIRE].

[36] S. Bose, L. Parker and Y. Peleg, Hawking radiation and unitary evolution, Phys. Rev. Lett. 76 (1996) 861 [gr-qc/9508027] [inSPIRE].

[37] S. Bose, L. Parker and Y. Peleg, Predictability and semiclassical approximation at the onset of black hole formation, Phys. Rev. D 54 (1996) 7490 [hep-th/9606152] [INSPIRE].

[38] M. Fitkevich, D. Levkov and Y. Zenkevich, Exact solutions and critical chaos in dilaton gravity with a boundary, JHEP 04 (2017) 108 [arXiv: 1702.02576] [INSPIRE].

[39] A. Strominger, Les Houches lectures on black holes, hep-th/9501071 [INSPIRE].

[40] C. Holzhey, F. Larsen and F. Wilczek, Geometric and renormalized entropy in conformal field theory, Nucl. Phys. B 424 (1994) 443 [hep-th/9403108] [INSPIRE].

[41] E. Bianchi, T. De Lorenzo and M. Smerlak, Entanglement entropy production in gravitational collapse: covariant regularization and solvable models, JHEP 06 (2015) 180 [arXiv: 1409.0144] [INSPIRE].

[42] M.R.R. Good, K. Yelshibekov and Y.C. Ong, On horizonless temperature with an accelerating mirror, JHEP 03 (2017) 013 [arXiv:1611.00809] [INSPIRE].

[43] A. Ashtekar, F. Pretorius and F.M. Ramazanoglu, Surprises in the evaporation of 2-dimensional black holes, Phys. Rev. Lett. 106 (2011) 161303 [arXiv:1011.6442] [INSPIRE].

[44] A. Ashtekar, F. Pretorius and F.M. Ramazanoglu, Evaporation of 2-dimensional black holes, Phys. Rev. D 83 (2011) 044040 [arXiv: 1012.0077] [inSPIRE].

[45] D. Grumiller, W. Kummer and D.V. Vassilevich, Dilaton gravity in two-dimensions, Phys. Rept. 369 (2002) 327 [hep-th/0204253] [INSPIRE].

[46] H. Afshar, H.A. González, D. Grumiller and D. Vassilevich, Flat space holography and the complex Sachdev-Ye-Kitaev model, Phys. Rev. D 101 (2020) 086024 [arXiv:1911.05739] [INSPIRE].

[47] D.V. Eremeev, Dilaton gravity with a boundary (in Russian), M.Sc. thesis, Moscow State University, Russia (2017).

[48] G.W. Gibbons and S.W. Hawking, Action Integrals and Partition Functions in Quantum Gravity, Phys. Rev. D 15 (1977) 2752 [INSPIRE].

[49] E. Poisson, A relativist's toolkit. the mathematics of black-hole mechanics, Cambridge University Press, Cambridge U.K. (2004). 
[50] P.C.W. Davies and S.A. Fulling, Radiation from a moving mirror in two-dimensional space-time conformal anomaly, Proc. Roy. Soc. Lond. A A 348 (1976) 393 [inSPIRE].

[51] F. Wilczek, Quantum purity at a small price: easing a black hole paradox, hep-th/9302096 [INSPIRE].

[52] E. Bianchi and M. Smerlak, Entanglement entropy and negative energy in two dimensions, Phys. Rev. D 90 (2014) 041904 [arXiv: 1404.0602] [INSPIRE].

[53] E. Bianchi and M. Smerlak, Last gasp of a black hole: unitary evaporation implies non-monotonic mass loss, Gen. Rel. Grav. 46 (2014) 1809 [arXiv:1405.5235] [INSPIRE].

[54] M.R.R. Good, E.V. Linder and F. Wilczek, Moving mirror model for quasithermal radiation fields, Phys. Rev. D 101 (2020) 025012 [arXiv: 1909.01129] [INSPIRE].

[55] A. Anderson and B.S. DeWitt, Does the topology of space fluctuate?, Found. Phys. 16 (1986) 91 [INSPIRE].

[56] A. Ishibashi and A. Hosoya, Naked singularity and thunderbolt, Phys. Rev. D 66 (2002) 104016 [gr-qc/0207054] [INSPIRE].

[57] T.M. Fiola, J. Preskill, A. Strominger and S.P. Trivedi, Black hole thermodynamics and information loss in two-dimensions, Phys. Rev. D 50 (1994) 3987 [hep-th/9403137] [INSPIRE].

[58] R.C. Myers, Black hole entropy in two-dimensions, Phys. Rev. D 50 (1994) 6412 [hep-th/9405162] [INSPIRE].

[59] M. Fitkevich, D. Levkov and S. Sibiryakov, Semiclassical $\mathcal{S}$-matrix and black hole entropy in dilaton gravity, arXiv:2006.03606 [INSPIRE].

[60] S.N. Solodukhin, Two-dimensional quantum corrected eternal black hole, Phys. Rev. D 53 (1996) 824 [hep-th/9506206] [INSPIRE].

[61] J.D. Hayward, Entropy in the RST model, Phys. Rev. D 52 (1995) 2239 [gr-qc/9412065] [INSPIRE].

[62] F. Bezrukov, D. Levkov and S. Sibiryakov, Semiclassical S-matrix for black holes, JHEP 12 (2015) 002 [arXiv: 1503.07181] [INSPIRE].

[63] I. Affleck, On constrained instantons, Nucl. Phys. B 191 (1981) 429 [inSPIRE].

[64] D.G. Levkov, A.G. Panin and S.M. Sibiryakov, Unstable semiclassical trajectories in tunneling, Phys. Rev. Lett. 99 (2007) 170407 [arXiv:0707.0433] [INSPIRE].

[65] V.A. Rubakov and M.E. Shaposhnikov, Electroweak baryon number nonconservation in the early universe and in high-energy collisions, Usp. Fiz. Nauk 166 (1996) 493 [hep-ph/9603208] [INSPIRE].

[66] M.K. Parikh and F. Wilczek, Hawking radiation as tunneling, Phys. Rev. Lett. 85 (2000) 5042 [hep-th/9907001] [INSPIRE].

[67] O. Klein, Die Reflexion von Elektronen an einem Potentialsprung nach der relativistischen Dynamik von Dirac, Z. Phys. 53 (1929) 157 [inSPIRE]. 